\title{
Heck-Suzuki Tandem Reaction for the Synthesis of 3-Benzazepines
}

\author{
2 Anatoly A. Peshkov, ${ }^{\dagger}$ Vsevolod A. Peshkov, ${ }^{*}{ }^{\dagger}, \S$ Olga P. Pereshivko, ${ }^{\dagger}$ Kristof Van Hecke, ${ }^{\dagger}$ \\ ${ }_{3}$ Rakesh Kumar, ${ }^{\dagger}, \#$ and Erik V. Van der Eycken*, ${ }^{\dagger}$ \\ $4{ }^{\dagger}$ Laboratory for Organic \& Microwave-Assisted Chemistry (LOMAC), Department of Chemistry, University of Leuven (KU Leuven), \\ 5 Celestijnenlaan 200F, B-3001 Leuven, Belgium \\ $6{ }^{\ddagger}$ Department of Inorganic and Physical Chemistry, Gent University, Krijgslaan 281 S3, B-9000 Gent, Belgium
}

7 S Supporting Information

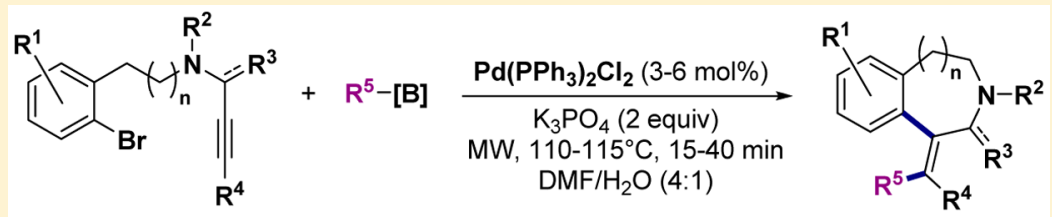

8 ABSTRACT: A novel procedure for the Heck-Suzuki tandem reaction suitable for the construction of nitrogen-containing 9 medium rings was developed to provide access toward the 3-benzazepine framework.

10

11 The 3-benzazepine core is present in a large variety of natural 12 products and important pharmaceuticals and might be rightfully 13 regarded as a privileged structure. ${ }^{1}$ Therefore, it has served as a 14 target motif for a large number of synthetic studies. Successful 15 examples include various types of ring-expansion reactions, ${ }^{2}$ 16 insertion of allenes into the $\mathrm{Pd}-\mathrm{C}$ bond of ortho-palladated 17 phenethylamines, ${ }^{3}$ and heterocyclizations involving either intra18 molecular reductive amination ${ }^{4}$ or transition-metal-catalyzed 19 triple-bond hydroamination. ${ }^{5}$ Radical, $^{6}$ Friedel-Crafts, ${ }^{7}$ and 20 Heck-type $^{8}$ carbocyclizations are also among the most applied 21 methodologies for the 3-benzazepine assembly.

22 In 1994, Tietze and Schimpf described an efficient route 23 toward 3-benzazepines starting from propargylamides contain24 ing an aryl iodide moiety by applying an intramolecular 25 version of the reductive Heck reaction that is also referred to as 26 formal triple bond hydroaryation. 9,10 Later on, our group 27 established a more general protocol that utilizes readily 28 accessible propargylamides derived from 3-substituted propiolic 29 acids and $o$-bromophenethylamines. ${ }^{11}$ Subsequently, we have 30 expanded this approach to the use of Ugi reaction derived 31 propargylamides ${ }^{12}$ and $\mathrm{A}^{3}$-coupling derived propargylamines ${ }^{13}$ 32 aiming to introduce an additional diversity in the resulting 33 3-benzazepines.'

34 These reductive Heck approaches operate through the cyclic 35 intermediate $\mathbf{A}$, resulting from the oxidative addition of aryl 36 bromide to the $\operatorname{Pd}(0)$-species and subsequent triple bond 37 insertion, which then undergoes the reduction with HCOONa 38 (sodium formate) into the 3-benzazepine of type 2. Trapping 39 this intermediate with an organoboron reagent 3 should 40 hypothetically result in the formation of the 3-benzazepine of 41 type 4 (Scheme 1). Such Heck-Suzuki tandem reactions are 42 well-known in the literature and have been previously 43 demonstrated to be highly efficient for the assembly of five44 and six-membered hetero- ${ }^{14}$ and alicycles. ${ }^{15}$ Moreover, this
Scheme 1. Synthesis of the 3-Benzazepine Framework through Palladium-Catalyzed Carbocyclizations of Propargylic Precursors

Previous work

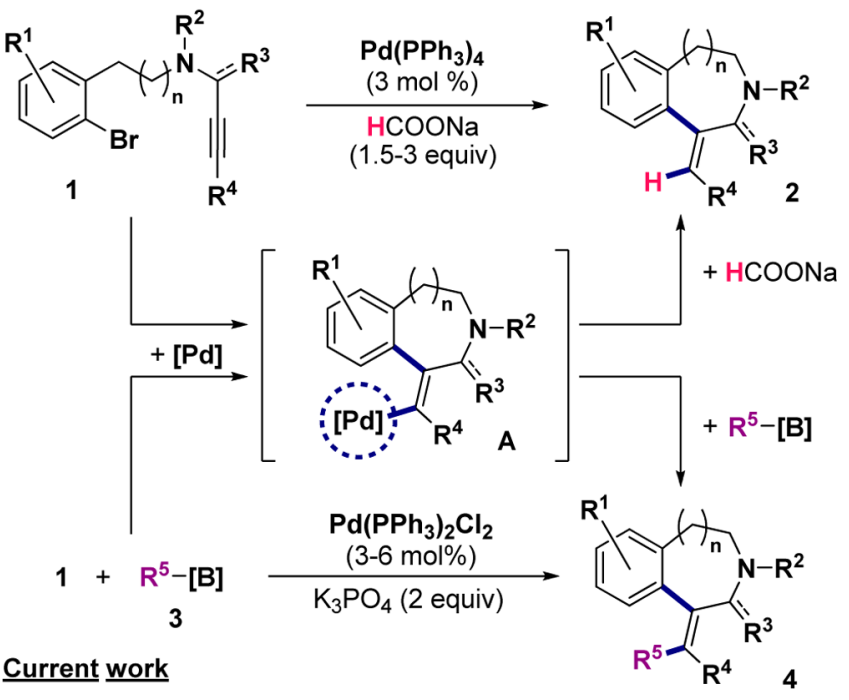

strategy proved to be useful for the synthesis of seven- 45 membered dibenzoxapine derivatives. ${ }^{16}$ However, to the best of 46 our knowledge, no general protocol allowing the application of 47 this process for the synthesis of nitrogen-containing medium- 48 rings is known in the literature. In order to fill this gap we 49 aimed to examine the Heck-Suzuki tandem reaction for the 50 synthesis and further diversification of the 3-benzazepine 51 scaffold. Herein we present the detailed studies on the scope 52 and limitations of the resulting procedure.

Received: March 25, 2015 
Table 1. Screening of Parameters for the Model Reaction of $1 \mathrm{a}$ and $3 \mathrm{a}^{a}$<smiles>CC#CC(=O)N(CCc1cc(OC)c(OC)cc1Br)C(C)(C)C</smiles>

$1 a$

\begin{tabular}{cc} 
entry & $x$ \\
\hline 1 & 1.5 \\
2 & 1.5 \\
3 & 1.2 \\
4 & 1.5 \\
5 & 1.5 \\
6 & 1.5 \\
7 & 1.5 \\
8 & 1.5 \\
9 & 1.5 \\
10 & 1.5 \\
11 & 1.8 \\
12 & 1.5 \\
13 & 1.5 \\
$\mathbf{1 4}$ & $\mathbf{1 . 5}$ \\
15 & 1.5 \\
16 & 1.5 \\
17 & 1.5 \\
18 & 1.5 \\
19 & 1.5 \\
20 & 1.5 \\
\hline
\end{tabular}

$\mathrm{PhB}(\mathrm{OH})_{2}$

(x equiv)

3a

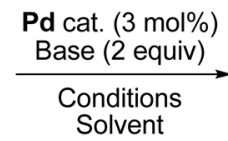

Solvent

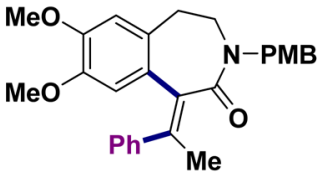

$4 a$

\begin{tabular}{|c|c|c|c|c|c|}
\hline Pd cat. & base & conditions & solvent & yield $^{b}(\%)$ & conversion $^{b}(\%)$ \\
\hline $\mathrm{Pd}\left(\mathrm{PPh}_{3}\right)_{4}$ & $\mathrm{~K}_{3} \mathrm{PO}_{4}$ & $\mathrm{MW}, 110^{\circ} \mathrm{C}, 15 \mathrm{~min}$ & DMF & 13 & 70 \\
\hline $\mathrm{Pd}\left(\mathrm{PPh}_{3}\right)_{4}$ & $\mathrm{~K}_{3} \mathrm{PO}_{4}$ & $\mathrm{MW}, 110^{\circ} \mathrm{C}, 15 \mathrm{~min}$ & $\mathrm{DMF} / \mathrm{H}_{2} \mathrm{O}(3: 1)$ & 31 & 100 \\
\hline $\mathrm{Pd}\left(\mathrm{PPh}_{3}\right)_{4}$ & $\mathrm{~K}_{3} \mathrm{PO}_{4}$ & $\mathrm{MW}, 110^{\circ} \mathrm{C}, 15 \mathrm{~min}$ & $\mathrm{DMF} / \mathrm{H}_{2} \mathrm{O}(3: 1)$ & 16 & 95 \\
\hline $\mathrm{Pd}\left(\mathrm{PPh}_{3}\right)_{4}$ & KOAc & $\mathrm{MW}, 110^{\circ} \mathrm{C}, 15 \mathrm{~min}$ & $\mathrm{DMF} / \mathrm{H}_{2} \mathrm{O}(3: 1)$ & 48 & 86 \\
\hline $\mathrm{Pd}\left(\mathrm{PPh}_{3}\right)_{4}$ & $\mathrm{Na}_{2} \mathrm{CO}_{3}$ & $\mathrm{MW}, 110^{\circ} \mathrm{C}, 15 \mathrm{~min}$ & $\mathrm{DMF} / \mathrm{H}_{2} \mathrm{O}(3: 1)$ & 30 & 100 \\
\hline $\mathrm{Pd}\left(\mathrm{PPh}_{3}\right)_{4}$ & $\mathrm{Cs}_{2} \mathrm{CO}_{3}$ & $\mathrm{MW}, 110^{\circ} \mathrm{C}, 15 \mathrm{~min}$ & $\mathrm{DMF} / \mathrm{H}_{2} \mathrm{O}(3: 1)$ & 21 & 100 \\
\hline $\mathrm{Pd}\left(\mathrm{PPh}_{3}\right)_{4}$ & $t \mathrm{BuOK}$ & $\mathrm{MW}, 110^{\circ} \mathrm{C}, 15 \mathrm{~min}$ & $\mathrm{DMF} / \mathrm{H}_{2} \mathrm{O}(3: 1)$ & 16 & 100 \\
\hline $\mathrm{Pd}\left(\mathrm{PPh}_{3}\right)_{2} \mathrm{Cl}_{2}$ & KOAc & $\mathrm{MW}, 110^{\circ} \mathrm{C}, 15 \mathrm{~min}$ & $\mathrm{DMF} / \mathrm{H}_{2} \mathrm{O}(3: 1)$ & 36 & 90 \\
\hline $\mathrm{Pd}\left(\mathrm{PPh}_{3}\right)_{2} \mathrm{Cl}_{2}$ & $\mathrm{~K}_{3} \mathrm{PO}_{4}$ & $\mathrm{MW}, 110^{\circ} \mathrm{C}, 15 \mathrm{~min}$ & $\mathrm{DMF} / \mathrm{H}_{2} \mathrm{O}(3: 1)$ & 55 & 100 \\
\hline $\mathrm{Pd}(\mathrm{OAc})_{2} / \mathrm{PPh}_{3}$ & $\mathrm{KOAc}$ & $\mathrm{MW}, 110^{\circ} \mathrm{C}, 15 \mathrm{~min}$ & $\mathrm{DMF} / \mathrm{H}_{2} \mathrm{O}(3: 1)$ & 41 & 88 \\
\hline $\mathrm{Pd}\left(\mathrm{PPh}_{3}\right)_{2} \mathrm{Cl}_{2}$ & $\mathrm{~K}_{3} \mathrm{PO}_{4}$ & $\mathrm{MW}, 110^{\circ} \mathrm{C}, 15 \mathrm{~min}$ & $\mathrm{DMF} / \mathrm{H}_{2} \mathrm{O}(3: 1)$ & 48 & 100 \\
\hline $\mathrm{Pd}\left(\mathrm{PPh}_{3}\right)_{2} \mathrm{Cl}_{2}$ & $\mathrm{~K}_{3} \mathrm{PO}_{4}$ & $\mathrm{MW}, 110^{\circ} \mathrm{C}, 15 \mathrm{~min}$ & $\mathrm{DMF} / \mathrm{H}_{2} \mathrm{O}(1: 1)$ & 17 & 100 \\
\hline $\mathrm{Pd}\left(\mathrm{PPh}_{3}\right)_{2} \mathrm{Cl}_{2}$ & $\mathrm{~K}_{3} \mathrm{PO}_{4}$ & $\mathrm{MW}, 110^{\circ} \mathrm{C}, 15 \mathrm{~min}$ & DMA $/ \mathrm{H}_{2} \mathrm{O}(3: 1)$ & 50 & 100 \\
\hline $\mathbf{P d}\left(\mathbf{P P h}_{3}\right)_{2} \mathrm{Cl}_{2}$ & $\mathrm{~K}_{3} \mathrm{PO}_{4}$ & $\mathrm{MW}, 110^{\circ} \mathrm{C}, 15 \mathrm{~min}$ & $\mathrm{DMF} / \mathrm{H}_{2} \mathrm{O}(4: 1)$ & $58(51)^{c}$ & 100 \\
\hline $\mathrm{Pd}(\mathrm{OAc})_{2} / \mathrm{PPh}_{3}$ & $\mathrm{~K}_{3} \mathrm{PO}_{4}$ & $\mathrm{MW}, 110^{\circ} \mathrm{C}, 15 \mathrm{~min}$ & $\mathrm{DMF} / \mathrm{H}_{2} \mathrm{O}(4: 1)$ & 43 & 100 \\
\hline $\mathrm{Pd}_{2} \mathrm{dba}_{3} / \mathrm{PPh}_{3}$ & $\mathrm{~K}_{3} \mathrm{PO}_{4}$ & $\mathrm{MW}, 110^{\circ} \mathrm{C}, 15 \mathrm{~min}$ & $\mathrm{DMF} / \mathrm{H}_{2} \mathrm{O}(4: 1)$ & 37 & 100 \\
\hline $\mathrm{Pd}\left(\mathrm{PPh}_{3}\right)_{2} \mathrm{Cl}_{2}$ & $\mathrm{~K}_{3} \mathrm{PO}_{4}$ & $\mathrm{MW}, 90{ }^{\circ} \mathrm{C}, 25 \mathrm{~min}$ & $\mathrm{DMF} / \mathrm{H}_{2} \mathrm{O}(4: 1)$ & 44 & 94 \\
\hline $\mathrm{Pd}\left(\mathrm{PPh}_{3}\right)_{2} \mathrm{Cl}_{2}$ & $\mathrm{~K}_{3} \mathrm{PO}_{4}$ & $\mathrm{MW}, 120^{\circ} \mathrm{C}, 10 \mathrm{~min}$ & $\mathrm{DMF} / \mathrm{H}_{2} \mathrm{O}(4: 1)$ & 31 & 88 \\
\hline $\mathrm{Pd}\left(\mathrm{PPh}_{3}\right)_{2} \mathrm{Cl}_{2}$ & $\mathrm{~K}_{3} \mathrm{PO}_{4}$ & oil bath, $90^{\circ} \mathrm{C}, 2 \mathrm{~h}$ & $\mathrm{DMF} / \mathrm{H}_{2} \mathrm{O}(4: 1)$ & 39 & 100 \\
\hline $\mathrm{Pd}\left(\mathrm{PPh}_{3}\right)_{2} \mathrm{Cl}_{2}$ & $\mathrm{~K}_{3} \mathrm{PO}_{4}$ & oil bath, $110^{\circ} \mathrm{C}, 1 \mathrm{~h}$ & DMF/water(4:1) & 35 & 100 \\
\hline
\end{tabular}

${ }^{a}$ The reactions were run on a $0.2 \mathrm{mmol}$ scale in $2.25 \mathrm{~mL}$ of solvent. ${ }^{b}$ Yields and conversions were determined by ${ }^{1} \mathrm{H}$ NMR using $3,4,5$ trimethoxybenzaldehyde as internal standard. ${ }^{c}$ Isolated yield is given in parentheses.

Table 2. Comparison of the Reactivity of Different Organoboron Reagents $3^{a}$
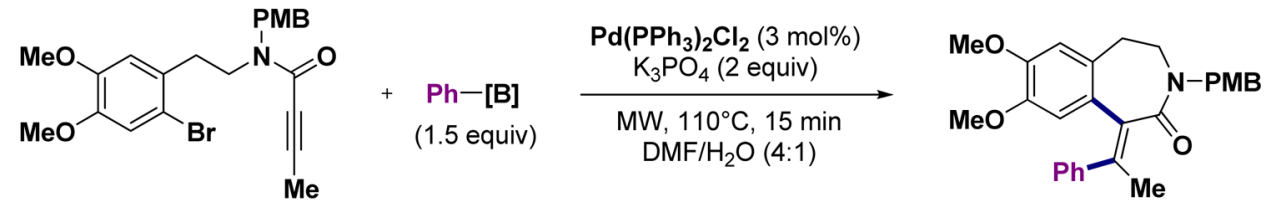

$1 a$

3

4a

\begin{tabular}{|c|c|c|c|}
\hline Entry & $\mathrm{Ph}-[\mathrm{B}] \quad(3)$ & Yield $^{b}$ & Conversion $^{b}$ \\
\hline 1 & $\mathrm{PhB}(\mathrm{OH})_{2} \quad 3 \mathrm{a}$ & $58(51)^{\mathrm{c}}$ & 100 \\
\hline 2 & $\mathrm{PhBF}_{3} \mathrm{~K} \quad 3 \mathbf{b}$ & 29 & 100 \\
\hline 3 & & $52(45)^{\mathrm{c}}$ & 100 \\
\hline 4 & $\mathrm{Ph}^{\prime}$ & 14 & 34 \\
\hline
\end{tabular}

${ }^{a_{1}}$ The reactions were run on a $0.2 \mathrm{mmol}$ scale in $2.25 \mathrm{~mL}$ of solvent. ${ }^{b}$ Yields and conversions were determined by ${ }^{1} \mathrm{H}$ NMR using $3,4,5$ trimethoxybenzaldehyde as internal standard. ${ }^{c}$ Isolated yields are given in parentheses.

\section{RESULTS AND DISCUSSION}

55 The reaction conditions for the Heck-Suzuki tandem process 56 were adjusted using propargylamide $1 \mathrm{a}$ and phenylboronic acid 57 3a as model substrates (Table 1$). \mathrm{Pd}\left(\mathrm{PPh}_{3}\right)_{4}$-catalyzed reaction 58 of $1 \mathrm{a}$ with 1.5 equiv of $3 \mathrm{a}$ and $\mathrm{K}_{3} \mathrm{PO}_{4}$ as a base conducted 59 under microwave irradiation at $110{ }^{\circ} \mathrm{C}$ for $15 \mathrm{~min}$ in $\mathrm{DMF}$ 60 resulted in $70 \%$ conversion of 1 a but gave only $13 \%$ yield of 61 the desired 3-benzazepine $\mathbf{4 a}$ as determined by ${ }^{1} \mathrm{H}$ NMR of the crude material after workup (Table 1, entry 1). Switching to 62 a DMF/water (3:1) mixture as solvent system led to full 63 conversion of $\mathbf{1 a}$ and an improved $31 \%$ yield for $4 \mathbf{a}$ (Table 1, 64 entry 2). An attempt to decrease the excess phenylboronic acid 65 3a resulted in incomplete conversion of 1a (Table 1, entry 3). 66 Next, we screened various bases in combination with different 67 $\mathrm{Pd}$ catalysts (Table 1, entries 4-10). The $\mathrm{Pd}\left(\mathrm{PPh}_{3}\right)_{2} \mathrm{Cl}_{2} / \mathrm{K}_{3} \mathrm{PO}_{4} 68$ combination was found to be the best, delivering target $4 \mathbf{a}$ in a 69 
Table 3. Scope and Limitations of the Heck-Suzuki Tandem Reaction Procedure for the Synthesis of 3-Benzazepine and 3-Benzazocine Frameworks ${ }^{a}$

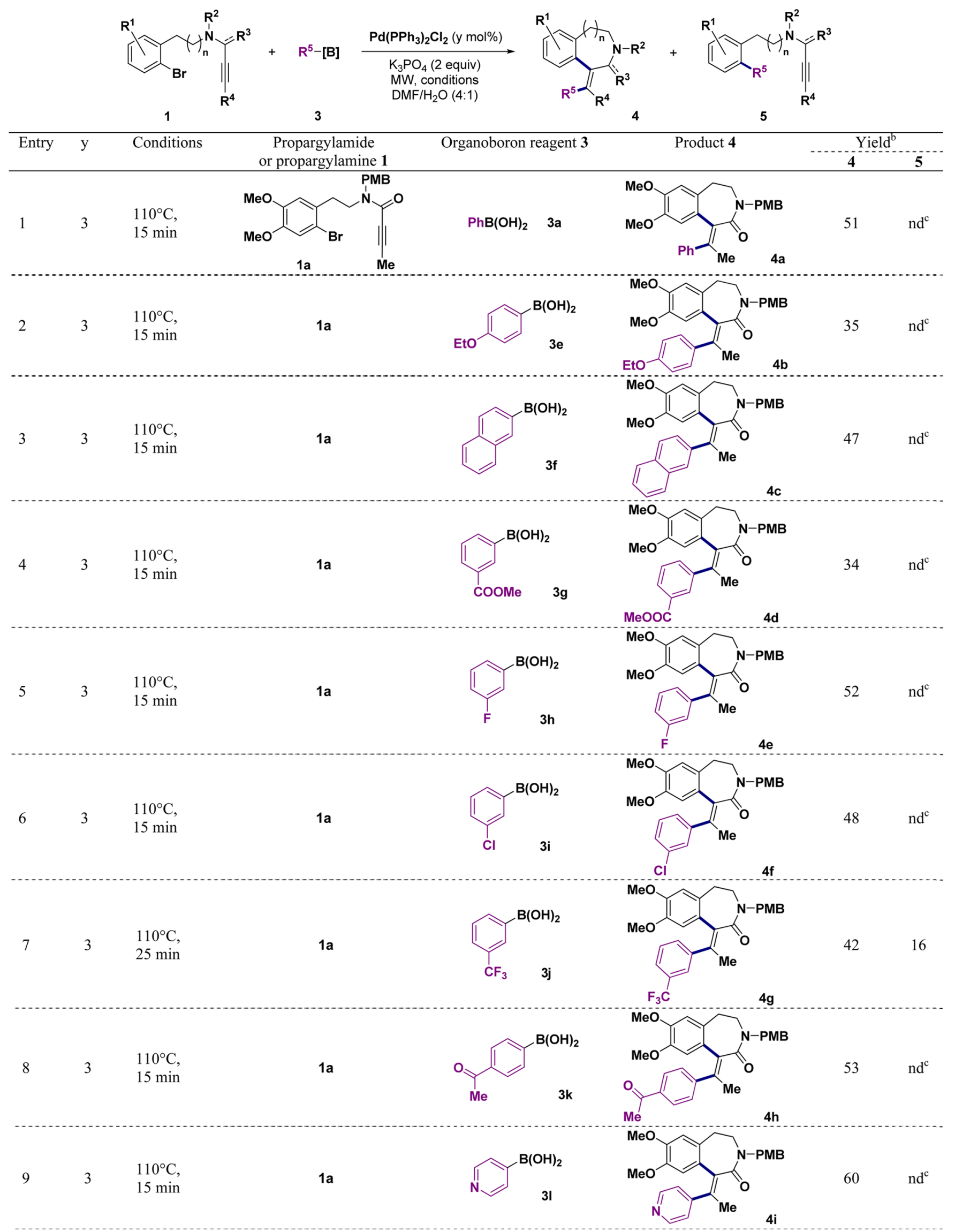


Table 3. continued

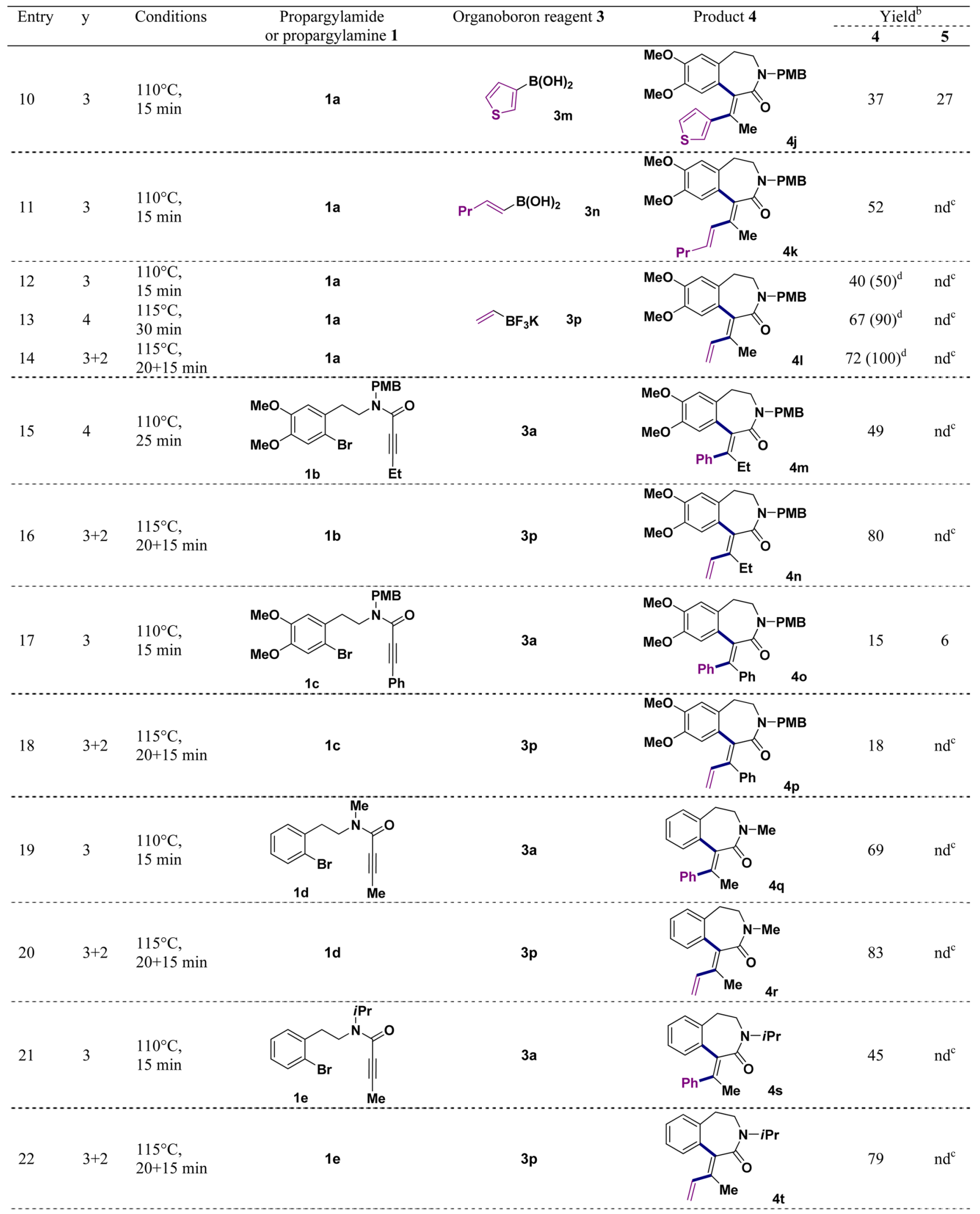


Table 3. continued

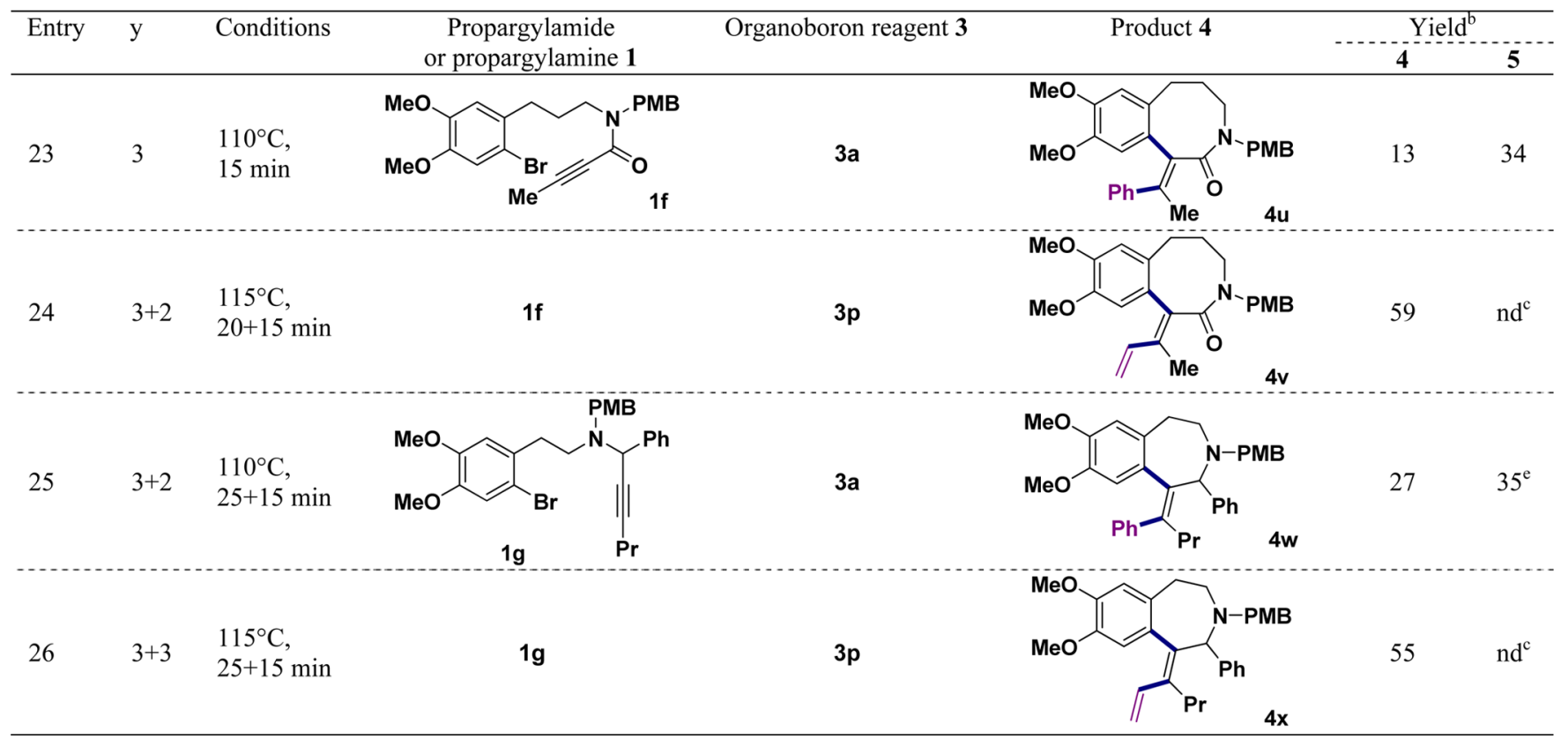

${ }^{a}$ The reactions were run on a $0.2 \mathrm{mmol}$ scale in $2.25 \mathrm{~mL}$ of solvent. ${ }^{b}$ Isolated yields. ${ }^{c}$ nd $=$ not detected or difficult to determine clearly. ${ }^{d}$ Conversions are given in parentheses. ${ }^{e}$ Yield was determined by ${ }^{1} \mathrm{H}$ NMR using 3,4,5-trimethoxybenzaldehyde as internal standard.

70 good NMR yield of 55\% (Table 1, entry 9). Interestingly, 71 increasing the excess phenylboronic acid 3 a up to 1.8 equiv did 72 not provide a better yield of $\mathbf{4 a}$ (Table 1, entry 11). Changing 73 the DMF-water ratio to $1: 1$ led to a significant drop in the 74 yield of $4 \mathrm{a}$ (Table 1, entry 12 ). The reaction in a DMA/water 75 (3:1) mixture gave a slightly decreased yield of 4 a compared to 76 the analogous reaction in DMF/water (3:1) (Table 1, entry 13 77 versus entry 9). Finally, reaction in DMF/water (4:1) delivered 78 3-benzazepine $4 \mathrm{a}$ in a best observed NMR yield of $58 \%$, which 79 corresponds to $51 \%$ isolated yield after column chromatography 80 and recrystallization from diethyl ether (Table 1, entry 14). 81 Further attempts to increase the yield of $\mathbf{4 a}$ by tuning the 82 catalytic system were unproductive (Table 1, entries 15 and 16). 83 In addition, no further improvement was achieved by changing 84 the reaction temperature as well as by applying conventional oil 85 bath heating (Table 1, entries 17-20).

86 We then decided to compare the reactivity of several common 87 phenylorganoboron reagents $\mathbf{3 a}-\mathbf{d}$ in the model reaction with 88 1a (Table 2). Phenylboronic acid 3a was found to be the most 89 efficient coupling partner (Table 2 , entry 1 ). Nonetheless, the 90 use of potassium phenyltrifluoroborate $\mathbf{3 b}$ and phenylboronic 91 acid pinacol ester $3 \mathbf{c}$ also resulted in a full conversion of $\mathbf{1 a}$, 92 although the yields for the desired 3-benzazepine 4a were 93 lower in these cases (Table 2, entries 2 and 3 versus entry 1). 94 The application of phenylboronic acid MIDA ester $3 \mathbf{d}$ gave a 95 very low conversion of $1 \mathbf{a}$ and as a result a poor yield for target 96 compound 4a (Table 2, entry 4).

97 Having these results in hand, we decided to evaluate the 98 scope and limitations of our procedure (Table 3 ). First we have 99 screened a number of aromatic $3 \mathbf{a}, \mathbf{e}-\mathbf{k}$ and heteroaromatic 100 31, $\mathbf{m}$ boronic acids in combination with propargylamide 1a. All 101 reactions were successful, delivering the desired 3-benzazepines $1024 \mathbf{a}-\mathbf{j}$ in up to $60 \%$ yield (Table 3 , entries $1-10$ ). The reaction 103 of 1 a with penten-1-ylboronic acid $3 \mathbf{n}$ also resulted in a good $10452 \%$ yield of 3-benzazepine $4 \mathbf{k}$ (Table 3, entry 11 ). At the 105 same time, the application of vinylpotassium trifluoroborate $3 p$ required some adjustments of the reaction conditions in 106 order to reach a full conversion of $\mathbf{1 a}$ but finally allowed to 107 obtain 3-benzazepine $\mathbf{4 1}$ in a very good yield of 72\% (Table 3, 108 entries 12-14). Next, we have screened the reactivity of various 109 propargylamides $\mathbf{1 b}-\mathbf{e}$ in combination with phenylboronic 110 acid $3 a$ and with vinylpotassium trifluoroborate $3 p$ (Table 3, 111 entries 15-22). Gratifyingly, the isolated yields of the desired 112 3-benzazepines in several cases have reached $80 \%$ yield. 113 However, in the reactions with propargylamide 1c derived 114 from phenylpropiolic acid, only very poor yields were obtained 115 (Table 3, entries 17 and 18). In the case of reactions with 116 propargylamide 1f, an expanded eight-membered ring could be 117 constructed although the yields for the resulting 3-benzazocines 118 $4 \mathbf{u}$ and $\mathbf{4 v}$ are significantly lower than for analogues 119 3-benzazepines $\mathbf{4 a}$ and $\mathbf{4 l}$ (Table 3, entries 23 and 24 versus 120 entries 1 and 14). Importantly, $\mathrm{A}^{3}$-coupling derived propargyl- 121 amine $1 \mathrm{~g}$ was also found to be applicable in this process 122 (Table 3, entries 25 and 26).

The reactions with vinylpotassium trifluoroborate $3 p$ in all 124 cases led to higher yields of the desired benzazepine and 125 benzazocine products compared to the analogues reactions with 126 phenylboronic acid 3a (Table 3, entries 14, 16, 18, 20, 22, 24, 127 and 26 versus entries 1, 15, 17, 19, 21, 23, and 25). This result 128 could be attributed to the smaller size of the introduced vinyl 129 fragment compared to the phenyl one.

130

In some cases, in addition to the desired Heck-Suzuki 131 product 4 the formation of byproduct 5 that results from the 132 direct Suzuki coupling of the aryl bromide moiety of $\mathbf{1}$ with 133 organoboron reagent $\mathbf{3}$ could be observed. In several instances, 134 such products 5 could be isolated and characterized (Table 3, 135 entries 7, 10, 17, 23, and 25).

136

Finally, we investigated the reactions of propargylamides 1a 137 and 1d with 2-phenyl-1-ethynylboronic acid pinacol ester 3o 138 (Table 4). Interestingly, in this case, in addition to the standard 139 product 4 another unexpected 3-benzazepine product $\mathbf{6}$ was 140 formed resulting from the double incorporation of organoboron 141 
Table 4. Heck-Suzuki Tandem Reaction with 2-Phenyl-1-ethynylboronic Acid Pinacol Ester 3o ${ }^{a}$<smiles>[R1]c1cccc(Br)c1CCN([R])C(=O)C#CC</smiles>

1

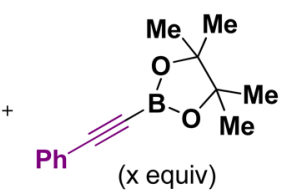

30


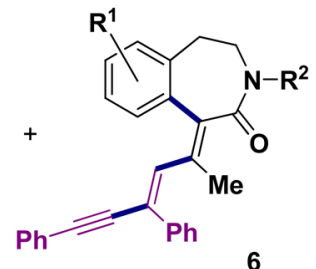

Entry $\quad$ Propargylamide 1130

${ }^{a}$ The reactions were run on a $0.2 \mathrm{mmol}$ scale in $2.25 \mathrm{~mL}$ of solvent. ${ }^{b}$ Isolated yields. ${ }^{c}$ Yields were determined by ${ }^{1} \mathrm{H}$ NMR using $3,4,5$ trimethoxybenzaldehyde as internal standard.

142 reagent 3o. We attempted to tune the 4 to 6 ratio by changing 143 the amounts of $3 \mathbf{0}$ added to the reaction; however, no 144 significant effect was achieved.

145 The structures of all prepared 3-benzazepines $\mathbf{4 a}-\mathbf{t}, \mathbf{w}-\mathbf{z}$ and 1466 and 3-benzazocines $4 \mathbf{u}, \mathbf{v}$ were confirmed by ${ }^{1} \mathrm{H}$ and ${ }^{13} \mathrm{C}$ 147 NMR spectroscopy and HRMS. In addition, the structures of 148 four representative products $\mathbf{4 m}, \mathbf{t}, \mathbf{z}$ and $\mathbf{6 b}$ were assured by 149 X-ray crystallographic analysis. ${ }^{17}$

\section{$150-$ CONCLUSION}

151 In summary, we have developed a novel protocol for the 152 tandem Heck-Suzuki reaction showing that it can be 153 successfully applied for the construction of nitrogen-containing 154 medium rings. Importantly, our methodology employs 155 propargylamides/amines comprising an aryl bromide function156 ality, while most of the known procedures leading to nitrogen157 containing five- and six-membered heterocycles ${ }^{14 a-c}$ generally 158 rely on more reactive aryl iodides. The adaptability of the 159 developed procedure toward the various organoboron sources 160 has also been evaluated.

\section{EXPERIMENTAL SECTION}

162 General Information. ${ }^{1} \mathrm{H}$ and ${ }^{13} \mathrm{C}$ NMR spectra were recorded at 163300 and $75 \mathrm{MHz}$, respectively. The ${ }^{1} \mathrm{H}$ and ${ }^{13} \mathrm{C}$ chemical shifts are 164 reported in parts per million relative to tetramethylsilane using the 165 residual solvent signal as the internal reference. High-resolution EI 166 mass spectra were recorded with a resolution of 10000 . The ion source 167 temperature was $150-250{ }^{\circ} \mathrm{C}$, as required. High-resolution ESI mass 168 spectra were acquired on a quadrupole orthogonal acceleration time169 of-flight mass spectrometer. Samples were infused at $3 \mu \mathrm{L} / \mathrm{min}$, and 170 spectra were obtained in positive-ionization mode with a resolution 171 of 15000 (fwhm) using leucine enkephalin as lock mass. Reversed172 phase HPLC separation was performed using a C18 $(5 \mu \mathrm{m} \times 150 \mathrm{~nm} \times$ $17322 \mathrm{~mm}$ ) preparative column.

174 The microwave reactions were carried out in $10 \mathrm{~mL}$ glass tubes and 175 sealed with a Teflon septum using a dedicated CEM-Discover mono176 mode microwave apparatus, operating with a frequency of $2.45 \mathrm{GHz}$.
The temperature of microwave reactions was measured by an inbuilt 177 infrared temperature probe.

Synthesis of the Starting Materials. The preparation procedures 179 and analytical data for compounds $1 \mathbf{1 a}, \mathbf{c}-\mathbf{f}^{11 \mathrm{a}}$ and $\mathbf{1 g}^{13}$ have been 180 described by us previously.

Synthesis of $\mathrm{N}$-(2-Bromo-4,5-dimethoxyphenethyl)-N-(4- 182 methoxybenzyl)pent-2-ynamide (1b). 2-(2-Bromo-4,5-dimethoxy- 183 phenyl)- $\mathrm{N}$-(4-methoxybenzyl)ethanamine (496 mg, $1.3 \mathrm{mmol}$ ) was 184 added in one portion to a mixture of pent-2-ynoic acid $(134 \mathrm{mg}, 185$ $1.37 \mathrm{mmol})$ and DCC (283 mg, $1.37 \mathrm{mmol})$ in dry DCM (6 mL). The 186 reaction mixture was stirred overnight at $\mathrm{rt}$. The subsequently formed 187 precipitate of $N, N^{\prime}$-dicyclohexylurea was filtered off and washed with 188 DCM. The combined organic layers were concentrated and subjected 189 to column chromatography on silica gel with EtOAc/heptane (3:7) as 190 eluent to deliver pure $\mathbf{1 b}$ as a 2:3 mixture of rotamers. Yield: $382 \mathrm{mg}, 191$ 64\%. Pale yellow oil. ${ }^{1} \mathrm{H}$ NMR (300 MHz, $\left.\mathrm{CDCl}_{3}\right): \delta 7.26-7.14(\mathrm{~m}, 192$ $2 \mathrm{H}), 6.99(\mathrm{~s}, 0.4 \mathrm{H}), 6.97(\mathrm{~s}, 0.6 \mathrm{H}), 6.90-6.81(\mathrm{~m}, 2 \mathrm{H}), 6.74(\mathrm{~s}, 0.6 \mathrm{H}), 193$ $6.56(\mathrm{~s}, 0.4 \mathrm{H}), 4.53(\mathrm{~s}, 2 \mathrm{H}), 3.87-3.76(\mathrm{~m}, 9 \mathrm{H}), 3.70-3.62(\mathrm{~m}, 0.8 \mathrm{H}), 194$ $3.50-3.41(\mathrm{~m}, 1.2 \mathrm{H}), 2.97-2.85(\mathrm{~m}, 2 \mathrm{H}), 2.43-2.30(\mathrm{~m}, 2 \mathrm{H}), 1.24-195$ $1.15(\mathrm{~m}, 3 \mathrm{H}) .{ }^{13} \mathrm{C}$ NMR $\left(75 \mathrm{MHz}, \mathrm{CDCl}_{3}\right): \delta 159.3,159.1,154.9,196$ $154.7,148.52,148.49,148.4,148.2,130.1,129.8,129.6,129.1,128.9,197$ 128.5, 115.5, 115.3, 114.3, 114.04, 113.96, 113.6, 113.5, 94.2, 93.9, 198 73.8, 73.4, 56.2, 56.11, 56.09, 55.3, 55.2, 52.8, 47.8, 47.2, 44.0, 35.0, 199 33.1, 12.93, 12.86, 12.8, 12.7. HRMS (EI): $\mathrm{m} / z[\mathrm{M}]^{+}$calcd for 200 $\mathrm{C}_{23} \mathrm{H}_{26} \mathrm{BrNO}_{4} 459.1045$, found 459.1059 .

General Procedure for the Heck-Suzuki Tandem Reaction 202 for the Synthesis of 3-Benzazepines $4 a-k, m, o, q, s, y, z$ and $6 a, b 203$ and benzazocine $4 \mathrm{u}$. $\mathrm{Pd}\left(\mathrm{PPh}_{3}\right)_{2} \mathrm{Cl}_{2}(4.2 \mathrm{mg}, 3 \mathrm{~mol} \%)$, organo- 204 boron reagent $3(0.3 \mathrm{mmol})$, and propargylamide $1(0.2 \mathrm{mmol}) 205$ were loaded into a microwave instrument vial and dissolved in DMF 206 $(1.8 \mathrm{~mL})$. Then $\mathrm{K}_{3} \mathrm{PO}_{4}(85 \mathrm{mg}, 0.4 \mathrm{mmol})$ was added followed by 207 distilled water $(0.45 \mathrm{~mL})$. The reaction vial was evacuated, flushed 208 with argon, sealed, and irradiated under stirring at a set temperature 209 of $110{ }^{\circ} \mathrm{C}$ for $15 \mathrm{~min}$ utilizing a maximum power of $100 \mathrm{~W}$. Upon 210 completion of the reaction, the vial was cooled with a stream of air. 211 The reaction mixture was diluted with EtOAc $(50 \mathrm{~mL})$, washed with 212 water $(2 \times 50 \mathrm{~mL})$, dried over $\mathrm{MgSO}_{4}$, and concentrated under 213 reduced pressure. The resulting crude material was subjected to the 214 appropriate purification procedure. 
216 (E)-7,8-Dimethoxy-3-(4-methoxybenzyl)-1-(1-phenylethylidene)217 4,5-dihydro-1H-benz[d]azepin-2(3H)-one (4a). The material 218 obtained after workup was subjected to column chromatography 219 on silica gel with heptane/EtOAc $(1: 1)$ as eluent followed by 220 recrystallization/washing with diethyl ether to deliver pure 4a. Yield: $22145 \mathrm{mg}, 51 \%$. White amorphous solid. ${ }^{1} \mathrm{H}$ NMR $\left(300 \mathrm{MHz}, \mathrm{CDCl}_{3}\right): \delta$ $2227.31-7.22(\mathrm{~m}, 2 \mathrm{H}), 7.20-7.05(\mathrm{~m}, 5 \mathrm{H}), 6.88(\mathrm{~d}, J=8.2 \mathrm{~Hz}, 2 \mathrm{H}), 6.45$ $223(\mathrm{~s}, 1 \mathrm{H}), 6.18(\mathrm{~s}, 1 \mathrm{H}), 5.02(\mathrm{~d}, J=14.8 \mathrm{~Hz}, 1 \mathrm{H}), 4.39-4.17(\mathrm{~m}, 2 \mathrm{H})$, $2243.81(\mathrm{~s}, 3 \mathrm{H}), 3.78(\mathrm{~s}, 3 \mathrm{H}), 3.39-3.25(\mathrm{~m}, 4 \mathrm{H}), 2.98-2.88(\mathrm{~m}, 2 \mathrm{H})$, $2252.33(\mathrm{~s}, 3 \mathrm{H}) .{ }^{13} \mathrm{C}$ NMR $\left(75 \mathrm{MHz}, \mathrm{CDCl}_{3}\right): \delta$ 171.6, 159.1, 147.9, $226146.5,141.9,137.5,134.6,129.7,129.4,128.6,128.1,127.7,126.7$, $227125.5,115.4,114.1,111.9,55.7,55.32,55.30,47.5,44.5,31.8$, 228 22.1. HRMS (EI): $m / z[M]^{+}$calcd for $\mathrm{C}_{28} \mathrm{H}_{29} \mathrm{NO}_{4} 443.2097$, found 229443.2096.

230 (E)-1-(1-(4-Ethoxyphenyl)ethylidene)-7,8-dimethoxy-3-(4-me231 thoxybenzyl)-4,5-dihydro-1H-benz[d]azepin-2(3H)-one (4b). The 232 material obtained after workup was subjected to column chromatog233 raphy on silica gel with heptane/EtOAc (1:1) as eluent followed by 234 recrystallization/washing with diethyl ether to deliver pure $\mathbf{4 b}$. Yield: $23534 \mathrm{mg}$, 35\%. White solid. Mp: $174-176{ }^{\circ} \mathrm{C} ;{ }^{1} \mathrm{H}$ NMR $(300 \mathrm{MHz}$, $\left.236 \mathrm{CDCl}_{3}\right): \delta 7.25(\mathrm{~d}, J=7.8 \mathrm{~Hz}, 2 \mathrm{H}), 7.01(\mathrm{~d}, J=8.3 \mathrm{~Hz}, 2 \mathrm{H}), 6.88(\mathrm{~d}$, $237 \mathrm{~J}=8.2 \mathrm{~Hz}, 2 \mathrm{H}), 6.68(\mathrm{~d}, J=8.3 \mathrm{~Hz}, 2 \mathrm{H}), 6.45(\mathrm{~s}, 1 \mathrm{H}), 6.23(\mathrm{~s}, 1 \mathrm{H})$, $2385.01(\mathrm{~d}, J=14.6 \mathrm{~Hz}, 1 \mathrm{H}), 4.37-4.16(\mathrm{~m}, 2 \mathrm{H}), 4.01-3.89(\mathrm{~m}, 2 \mathrm{H})$, $2393.81(\mathrm{~s}, 3 \mathrm{H}), 3.79(\mathrm{~m}, 3 \mathrm{H}), 3.38-3.26(\mathrm{~m}, 4 \mathrm{H}), 2.97-2.87(\mathrm{~m}, 2 \mathrm{H})$, $2402.30(\mathrm{~s}, 3 \mathrm{H}), 1.36(\mathrm{t}, J=6.9 \mathrm{~Hz}, 3 \mathrm{H}) ;{ }^{13} \mathrm{C}$ NMR $\left(75 \mathrm{MHz}, \mathrm{CDCl}_{3}\right)$ : $241 \delta 171.8,159.0,157.7,147.9,146.6,137.0,133.9,133.7,129.8,129.7$, $242129.4,127.6,125.8,115.4,114.1,114.0,111.9,63.3,55.7,55.4$, 243 55.3, 47.5, 44.5, 31.8, 22.0, 14.8; HRMS (EI): $m / z[\mathrm{M}]^{+}$calcd for $244 \mathrm{C}_{30} \mathrm{H}_{33} \mathrm{NO}_{5}$ 487.2359, found 487.2354.

245 (E)-7,8-dimethoxy-3-(4-methoxybenzyl)-1-(1-(naphthalen-2-yl)246 ethylidene)-4,5-dihydro-1H-benz[d]azepin-2(3H)-one (4c). The ma247 terial obtained after workup was subjected to column chromato248 graphy on silica gel with heptane/EtOAc (1:1) as eluent followed by 249 recrystallization/washing with diethyl ether to deliver pure 4c. Yield: $25046 \mathrm{mg}$, 47\%. Beige solid. Mp: 243-246 ${ }^{\circ} \mathrm{C} ;{ }^{1} \mathrm{H}$ NMR $(300 \mathrm{MHz}$, $\left.251 \mathrm{CDCl}_{3}\right): \delta 7.74-7.62(\mathrm{~m}, 3 \mathrm{H}), 7.57(\mathrm{~d}, J=8.5 \mathrm{~Hz}, 1 \mathrm{H}), 7.44-7.36$ $252(\mathrm{~m}, 2 \mathrm{H}), 7.28(\mathrm{~d}, J=8.6 \mathrm{~Hz}, 2 \mathrm{H}), 7.14(\mathrm{~d}, J=8.5 \mathrm{~Hz}, 1 \mathrm{H}), 6.89(\mathrm{~d}$, $253 J=8.0 \mathrm{~Hz}, 2 \mathrm{H}), 6.46(\mathrm{~s}, 1 \mathrm{H}), 6.22(\mathrm{~s}, 1 \mathrm{H}), 5.04(\mathrm{~d}, J=14.7 \mathrm{~Hz}, 1 \mathrm{H})$, $2544.43-4.25(\mathrm{~m}, 2 \mathrm{H}), 3.81(\mathrm{~s}, 3 \mathrm{H}), 3.76(\mathrm{~s}, 3 \mathrm{H}), 3.43-3.32(\mathrm{~m}, 1 \mathrm{H})$, $2553.07(\mathrm{~s}, 3 \mathrm{H}), 3.01-2.92(\mathrm{~m}, 2 \mathrm{H}), 2.42(\mathrm{~s}, 3 \mathrm{H}) ;{ }^{13} \mathrm{C}$ NMR $(75 \mathrm{MHz}$, $\left.256 \mathrm{CDCl}_{3}\right): \delta 171.6,159.1,148.0,146.5,139.5,137.3,135.0,133.2,132.1$, $257129.7,129.4,127.8,127.7,127.5,127.34,127.30,127.0,126.1,125.9$, 258 125.4, 115.5, 114.1, 112.0, 55.6, 55.3, 55.2, 47.5, 44.6, 31.9, 22.3; 259 HRMS (EI): $m / z[\mathrm{M}]^{+}$calcd for $\mathrm{C}_{32} \mathrm{H}_{31} \mathrm{NO}_{4}$ 493.2253, found 260493.2269.

261 (E)-methyl 3-(1-(7,8-dimethoxy-3-(4-methoxybenzyl)-2-oxo262 2,3,4,5-tetrahydro-1H-benz[d]azepin-1-ylidene)ethyl)benzoate 263 (4d). The material obtained after workup was subjected to column 264 chromatography on silica gel with heptane/EtOAc (1:1) as eluent 265 followed by recrystallization/washing with diethyl ether to deliver 266 pure 4 d. Yield: $34 \mathrm{mg}$, 34\%. Beige solid. Mp: $132-135{ }^{\circ} \mathrm{C}$. ${ }^{1} \mathrm{H}$ NMR $267\left(300 \mathrm{MHz} \mathrm{CDCl}_{3}\right): \delta 7.87-7.76(\mathrm{~m}, 2 \mathrm{H}), 7.31-7.16(\mathrm{~m}, 4 \mathrm{H}), 6.88$ $268(\mathrm{~d}, J=8.5 \mathrm{~Hz}, 2 \mathrm{H}), 6.46(\mathrm{~s}, 1 \mathrm{H}), 6.15$ (s. $1 \mathrm{H}), 4.99(\mathrm{~d}, J=14.7 \mathrm{~Hz}$, $2691 \mathrm{H}), 4.41-4.18(\mathrm{~m}, 2 \mathrm{H}), 3.87(\mathrm{~s}, 3 \mathrm{H}), 3.81(\mathrm{~s}, 3 \mathrm{H}), 3.78(\mathrm{~s}, 3 \mathrm{H})$, $2703.42-3.22(\mathrm{~m}, 4 \mathrm{H}), 3.01-2.84(\mathrm{~m}, 2 \mathrm{H}), 2.34(\mathrm{~s}, 3 \mathrm{H}) .{ }^{13} \mathrm{C} \mathrm{NMR}$ $271\left(75 \mathrm{MHz}, \mathrm{CDCl}_{3}\right): \delta 171.3,166.7,159.1,148.1,146.6,142.3,136.3$, $272135.6,133.3,130.1,129.7,129.6,129.4,128.1,128.0,127.9,124.9$, 273 115.2, 114.1, 112.1, 55.7, 55.4, 55.3, 52.1, 47.5, 44.6, 31.8, 22.0. HRMS 274 (ESI): $m / z[\mathrm{M}+\mathrm{H}]^{+}$calcd for $\mathrm{C}_{30} \mathrm{H}_{32} \mathrm{NO}_{6} 502.2224$, found 502.2218 . 275 (E)-1-(1-(3-Fluorophenyl)ethylidene)-7,8-dimethoxy-3-(4-me276 thoxybenzyl)-4,5-dihydro-1H-benz[d]azepin-2(3H)-one (4e). The 277 material obtained after workup was subjected to column chromatog278 raphy on silica gel with heptane/EtOAc (1:1) as eluent followed by 279 recrystallization/washing with diethyl ether to deliver pure 4e. Yield: $28048 \mathrm{mg}, 52 \%$. White solid. Mp: $163-165{ }^{\circ} \mathrm{C} .{ }^{1} \mathrm{H}$ NMR $(300 \mathrm{MHz}$, $\left.281 \mathrm{CDCl}_{3}\right): \delta 7.25(\mathrm{~d}, J=8.0 \mathrm{~Hz}, 2 \mathrm{H}), 7.13(\mathrm{q}, J=7.2 \mathrm{~Hz}, 1 \mathrm{H}), 6.92-$ $2826.76(\mathrm{~m}, 5 \mathrm{H}), 6.46(\mathrm{~s}, 1 \mathrm{H}), 6.20(\mathrm{~s}, 1 \mathrm{H}), 4.99(\mathrm{~d}, J=14.7 \mathrm{~Hz}, 1 \mathrm{H})$, $2834.34(\mathrm{~d}, J=14.7 \mathrm{~Hz}, 1 \mathrm{H}), 4.26-4.13(\mathrm{~m}, 1 \mathrm{H}), 3.81(\mathrm{~s}, 3 \mathrm{H}), 3.79(\mathrm{~s}$, $2843 \mathrm{H}), 3.39-3.29(\mathrm{~m}, 4 \mathrm{H}), 2.97-2.89(\mathrm{~m}, 2 \mathrm{H}), 2.30(\mathrm{~s}, 3 \mathrm{H}) .{ }^{13} \mathrm{C}$ NMR $285\left(75 \mathrm{MHz}, \mathrm{CDCl}_{3}\right): \delta 171.3,162.5(\mathrm{~d}, J=245.9 \mathrm{~Hz}), 159.1,148.2$,
146.6, $144.3(\mathrm{~d}, J=7.5 \mathrm{~Hz}), 136.1(\mathrm{~d}, J=1.9 \mathrm{~Hz}), 135.5,129.59,286$ 129.55 (d, $J=7.3 \mathrm{~Hz}), 129.4,127.8,125.0,124.4(\mathrm{~d}, J=2.8 \mathrm{~Hz}), 115.6287$ $(\mathrm{d}, J=21.6 \mathrm{~Hz}), 115.1,114.1,113.7(\mathrm{~d}, J=21.1 \mathrm{~Hz}), 112.1,55.7,288$ 55.4, 55.3, 47.5, 44.5, 31.8, 21.9. HRMS (EI): $\mathrm{m} / z[\mathrm{M}]^{+}$calcd for 289 $\mathrm{C}_{28} \mathrm{H}_{28} \mathrm{FNO}_{4} 461.2002$, found 461.2025 .

(E)-1-(1-(3-Chlorophenyl)ethylidene)-7,8-dimethoxy-3-(4-me- 291 thoxybenzyl)-4,5-dihydro-1H-benz[d]azepin-2(3H)-one (4f). The 292 material obtained after workup was subjected to column chromatog- 293 raphy on silica gel with heptane/EtOAc (1:1) as eluent followed by 294 recrystallization/washing with diethyl ether to deliver pure 4f. Yield: 295 $46 \mathrm{mg}, 48 \%$. White solid. Mp: $161-163{ }^{\circ} \mathrm{C} .{ }^{1} \mathrm{H}$ NMR (300 MHz, 296 $\left.\mathrm{CDCl}_{3}\right): \delta 7.25(\mathrm{~d}, J=8.6 \mathrm{~Hz}, 2 \mathrm{H}), 7.15-7.03(\mathrm{~m}, 3 \mathrm{H}), 6.98-6.92297$ $(\mathrm{m}, 1 \mathrm{H}), 6.88(\mathrm{~d}, J=8.6 \mathrm{~Hz}, 2 \mathrm{H}), 6.46(\mathrm{~s}, 1 \mathrm{H}), 6.19(\mathrm{~s}, 1 \mathrm{H}), 4.99(\mathrm{~d}, 298$ $J=14.7 \mathrm{~Hz}, 1 \mathrm{H}), 4.34(\mathrm{~d}, J=14.7 \mathrm{~Hz}, 1 \mathrm{H}), 4.26-4.12(\mathrm{~m}, 1 \mathrm{H}), 3.81299$ (s, 3H), $3.79(\mathrm{~s}, 3 \mathrm{H}), 3.39-3.29(\mathrm{~m}, 4 \mathrm{H}), 2.97-2.87(\mathrm{~m}, 2 \mathrm{H}), 2.29(\mathrm{~s}, 300$ $3 \mathrm{H}) .{ }^{13} \mathrm{C}$ NMR $\left(75 \mathrm{MHz}, \mathrm{CDCl}_{3}\right): \delta 171.2,159.1,148.2,146.6,143.9,301$ $136.0,135.7,133.9,129.6,129.4,129.3,128.6,127.8,126.89,126.86,302$ 124.9, 115.2, 114.1, 112.1, 55.7, 55.4, 55.3, 47.5, 44.5, 31.8, 22.0. 303 HRMS (EI): $m / z[\mathrm{M}]^{+}$calcd for $\mathrm{C}_{28} \mathrm{H}_{28} \mathrm{ClNO}_{4}$ 477.1707, found 304 477.1720 .

(E)-7,8-Dimethoxy-3-(4-methoxybenzyl)-1-(1-(3-306 (trifluoromethyl)phenyl)ethylidene)-4,5-dihydro-1H-benz[d]azepin- 307 $2(3 H)$-one $(\mathbf{4 g})$. The reaction time was $25 \mathrm{~min}$. The material obtained 308 after workup was subjected to column chromatography on silica gel 309 with heptane/EtOAc (1:1) as eluent followed by recrystallization from 310 diethyl ether to deliver pure $\mathbf{4 g}$. The mother liquor was evaporated and 311 subjected to reversed-phase preparative HPLC with gradient pump 312 mode, $\mathrm{MeCN} / \mathrm{H}_{2} \mathrm{O}$ with $0.1 \% \mathrm{HCOOH}(30-40-50-60-70-313$ $70-80-90-100 \%$, $10 \mathrm{~min}$ intervals) as eluent, and a flow rate of 314 $10 \mathrm{~mL} / \mathrm{min}$ to deliver a second portion of pure $4 \mathrm{~g}\left(t_{\mathrm{R}}=42 \mathrm{~min}\right) .315$ Combined yield: $43 \mathrm{mg}$, 42\%. White solid. Mp: $142-144{ }^{\circ} \mathrm{C} .{ }^{1} \mathrm{H} 316$ NMR (300 MHz, CDCl $\left.{ }_{3}\right): \delta 7.45-7.36(\mathrm{~m}, 2 \mathrm{H}), 7.31-7.19(\mathrm{~m}, 4 \mathrm{H}), 317$ $6.88(\mathrm{~d}, J=8.3 \mathrm{~Hz}, 2 \mathrm{H}), 6.46(\mathrm{~s}, 1 \mathrm{H}), 6.11(\mathrm{~s}, 1 \mathrm{H}), 5.00(\mathrm{~d}, J=14.7318$ $\mathrm{Hz}, 1 \mathrm{H}), 4.35(\mathrm{~d}, J=14.7 \mathrm{~Hz}, 1 \mathrm{H}), 2.30-2.15(\mathrm{~m}, 1 \mathrm{H}), 3.81(\mathrm{~s}, 3 \mathrm{H}), 319$ $3.78(\mathrm{~s}, 3 \mathrm{H}), 3.42-3.26(\mathrm{~m}, 4 \mathrm{H}), 2.98-2.88(\mathrm{~m}, 2 \mathrm{H}), 2.34(\mathrm{~s}, 3 \mathrm{H}) .320$ ${ }^{13} \mathrm{C}$ NMR $\left(75 \mathrm{MHz}, \mathrm{CDCl}_{3}\right): \delta 171.1,159.1,148.3,146.7,142.9,321$ 136.2, 135.9, 132.0, $130.6(\mathrm{q}, J=32.1 \mathrm{~Hz}), 129.6,129.4,128.6,128.0,322$ $125.4(\mathrm{q}, J=3.6 \mathrm{~Hz}), 123.9(\mathrm{~d}, J=272.2 \mathrm{~Hz}), 124.7,123.4(\mathrm{q}, J=4.1323$ $\mathrm{Hz}), 115.1,114.1,112.2,55.8,55.3,47.6,44.6,31.8,21.8$. HRMS (EI): 324 $m / z[\mathrm{M}]^{+}$calcd for $\mathrm{C}_{29} \mathrm{H}_{28} \mathrm{~F}_{3} \mathrm{NO}_{4} 511.1970$, found 511.1975. Further 325 elution provided $\mathrm{N}$-(2-(4,5-dimethoxy-3'-(trifluoromethyl)biphenyl-2- 326 yl)ethyl)- $\mathrm{N}$-(4-methoxybenzyl)but-2-ynamide (5g) as a $\sim 3: 2$ mixture 327 of rotamers $\left(t_{\mathrm{R}}=45 \mathrm{~min}\right)$. Yield: $16 \mathrm{mg}, 16 \%$. Pale yellow oil. ${ }^{1} \mathrm{H} 328$ NMR $\left(300 \mathrm{MHz}, \mathrm{CDCl}_{3}\right): \delta 7.70-7.40(\mathrm{~m}, 4 \mathrm{H}), 6.95-6.85(\mathrm{~m}, 2 \mathrm{H}), 329$ 6.84-6.72 (m, 2.6H), 6.72-6.68 (m, 0.8H), $6.67(\mathrm{~s}, 0.6 \mathrm{H}), 4.31(\mathrm{~s}, 330$ $1.2 \mathrm{H}), 4.17(\mathrm{~s}, 0.8 \mathrm{H}), 3.92(\mathrm{~s}, 1.8 \mathrm{H}), 3.91(\mathrm{~s}, 1.2 \mathrm{H}), 3.87(\mathrm{~s}, 1.2 \mathrm{H}), 331$ $3.86(\mathrm{~s}, 1.8 \mathrm{H}), 3.79(\mathrm{~s}, 1.8 \mathrm{H}), 3.77(\mathrm{~s}, 1.2 \mathrm{H}), 3.48-3.36(\mathrm{~m}, 0.8 \mathrm{H}), 332$ $3.30-3.17(\mathrm{~m}, 1.2 \mathrm{H}), 2.85-2.65(\mathrm{~m}, 2 \mathrm{H}), 1.97(\mathrm{~s}, 1.8 \mathrm{H}), 1.92(\mathrm{~s}, 333$ $1.2 \mathrm{H}) .{ }^{13} \mathrm{C}$ NMR $\left(75 \mathrm{MHz}, \mathrm{CDCl}_{3}\right): \delta 159.2,159.0,154.64,154.56,334$ $148.84,148.77,147.65,147.4,142.14,142.08,133.1,132.9,132.6,335$ $130.7(\mathrm{~m}), 129.5,128.9,128.7,128.6,128.52,128.45,128.2,128.0,336$ $126.1(\mathrm{~m}), 123.7(\mathrm{~m}), 114.0,113.9,113.2,113.0,112.9,112.8,89.0,337$ 88.6, 73.5, 73.4, 56.09, 56.05, 56.02, 55.3, 55.2, 52.3, 48.9, 46.6, 45.1, 338 31.6, 30.0, 4.0, 3.9. HRMS (EI): $m / z[\mathrm{M}]^{+}$calcd for $\mathrm{C}_{29} \mathrm{H}_{28} \mathrm{~F}_{3} \mathrm{NO}_{4} 339$ 511.1970 , found 511.1987 .

(E)-1-(1-(4-Acetylphenyl)ethylidene)-7,8-dimethoxy-3-(4-methox- 341 ybenzyl)-4,5-dihydro-1H-benz[d]azepin-2(3H)-one (4h). The materi- 342 al obtained after workup was subjected to column chromatography on 343 silica gel with heptane/EtOAc $(1: 1)$ as eluent followed by reversed- 344 phase preparative HPLC with gradient pump mode, $\mathrm{MeCN} / \mathrm{H}_{2} \mathrm{O} 345$ with $0.1 \% \mathrm{HCOOH}(30-40-50-60-70-70-80-90-100 \%, 10$ min 346 intervals) as eluent, and a flow rate of $10 \mathrm{~mL} / \mathrm{min}$ to deliver pure $4 \mathbf{h} 347$ $\left(t_{\mathrm{R}}=31 \mathrm{~min}\right)$. Yield: $51.5 \mathrm{mg}, 53 \%$. Yellow amorphous solid. ${ }^{1} \mathrm{H}$ NMR 348 $\left(300 \mathrm{MHz}, \mathrm{CDCl}_{3}\right): \delta 7.77(\mathrm{~d}, J=8.0 \mathrm{~Hz}, 2 \mathrm{H}), 7.26(\mathrm{~d}, J=8.3 \mathrm{~Hz}, 349$ $2 \mathrm{H}), 7.20(\mathrm{~d}, J=8.0 \mathrm{~Hz}, 2 \mathrm{H}), 6.88(\mathrm{~d}, J=8.3 \mathrm{~Hz}, 2 \mathrm{H}), 6.47(\mathrm{~s}, 1 \mathrm{H}), 350$ $6.15(\mathrm{~s}, 1 \mathrm{H}), 5.00(\mathrm{~d}, J=14.7 \mathrm{~Hz}, 1 \mathrm{H}), 4.35(\mathrm{~d}, J=14.7 \mathrm{~Hz}, 1 \mathrm{H}), 351$ 4.30-4.16 (m, 1H), $3.81(\mathrm{~s}, 3 \mathrm{H}), 3.79(\mathrm{~s}, 3 \mathrm{H}), 3.41-3.32(\mathrm{~m}, 1 \mathrm{H}), 352$ $3.28(\mathrm{~s}, 3 \mathrm{H}), 2.98-2.90(\mathrm{~m}, 2 \mathrm{H}), 2.53(\mathrm{~s}, 3 \mathrm{H}), 2.33(\mathrm{~s}, 3 \mathrm{H}) .{ }^{13} \mathrm{C}$ NMR 353 $\left(75 \mathrm{MHz}, \mathrm{CDCl}_{3}\right): \delta 197.4,171.1,159.1,148.3,147.2,146.6,136.3,354$ $136.1,135.3,129.6,129.4,128.9,128.1,127.9,125.0,115.2,114.1,355$ 
356 112.1, 55.7, 55.4, 55.3, 47.5, 44.5, 31.8, 26.5, 21.8. HRMS (EI): $m / z$ $357[\mathrm{M}]^{+}$calcd for $\mathrm{C}_{30} \mathrm{H}_{31} \mathrm{NO}_{5}$ 485.2202, found 485.2207.

358 (E)-7,8-Dimethoxy-3-(4-methoxybenzyl)-1-(1-(pyridin-4-yl)359 ethylidene)-4,5-dihydro- $1 \mathrm{H}$-benz[d]azepin-2(3H)-one (4i). The ma360 terial after workup was subjected to column chromatography on silica 361 gel with EtOAc as eluent followed by reversed-phase preparative 362 HPLC using gradient pump mode and $\mathrm{MeCN} / \mathrm{H}_{2} \mathrm{O}(20-30-30-$ $36340-100 \%, 10 \mathrm{~min}$ intervals) as eluent with a flow rate of $10 \mathrm{~mL} / \mathrm{min}$ 364 to deliver pure $4 \mathrm{i}\left(t_{\mathrm{R}}=37 \mathrm{~min}\right)$. Yield: $53 \mathrm{mg}, 60 \%$. Yellow oil. ${ }^{1} \mathrm{H}$ $365 \operatorname{NMR}\left(300 \mathrm{MHz}, \mathrm{CDCl}_{3}\right): \delta 8.42(\mathrm{bs}, 2 \mathrm{H}), 7.25(\mathrm{~d}, J=8.3 \mathrm{~Hz}, 2 \mathrm{H})$, $3667.08-7.00(\mathrm{~m}, 2 \mathrm{H}), 6.88(\mathrm{~d}, J=8.3 \mathrm{~Hz}, 2 \mathrm{H}), 6.49(\mathrm{~s}, 1 \mathrm{H}), 6.14(\mathrm{~s}$, $3671 \mathrm{H}), 4.97(\mathrm{~d}, J=14.7 \mathrm{~Hz}, 1 \mathrm{H}), 4.36(\mathrm{~d}, J=14.7 \mathrm{~Hz}, 1 \mathrm{H}), 4.26-4.12$ $368(\mathrm{~m}, 1 \mathrm{H}), 3.84-3.76(\mathrm{~m}, 6 \mathrm{H}), 3.42-3.30(\mathrm{~m}, 4 \mathrm{H}), 2.98-2.89(\mathrm{~m}, 2 \mathrm{H})$, $3692.31(\mathrm{~s}, 3 \mathrm{H}) .{ }^{13} \mathrm{C}$ NMR $\left(75 \mathrm{MHz}, \mathrm{CDCl}_{3}\right): \delta 170.8,159.2,150.5$, $370149.1,148.6,146.8,137.2,134.4,129.5,129.4,128.0,124.3,123.7$, $371114.9,114.1,112.3,55.7,55.5,55.3,47.6,44.5,31.8,21.3$. HRMS (EI): $372 \mathrm{~m} / z[\mathrm{M}]^{+}$calcd for $\mathrm{C}_{27} \mathrm{H}_{28} \mathrm{~N}_{2} \mathrm{O}_{4} 444.2049$, found 444.2065.

373 (E)-7,8-Dimethoxy-3-(4-methoxybenzyl)-1-(1-(thiophene-3-yl)374 ethylidene)-4,5-dihydro-1H-benz[d]azepin-2(3H)-one (4j). The ma375 terial obtained after workup was subjected to column chromatography 376 on silica gel with heptane/EtOAc (1:1) as eluent followed by reversed377 phase preparative HPLC with gradient pump mode, $\mathrm{MeCN} / \mathrm{H}_{2} \mathrm{O}$ with $3780.1 \% \mathrm{HCOOH}(30-40-50-60-60-70-70-100 \%, 10 \mathrm{~min}$ intervals) 379 as eluent, and a flow rate of $10 \mathrm{~mL} / \mathrm{min}$ to deliver pure $4 \mathbf{j}\left(t_{\mathrm{R}}=35 \mathrm{~min}\right)$. 380 Yield: $33 \mathrm{mg}$, 37\%. Yellow oil. ${ }^{1} \mathrm{H}$ NMR $\left(300 \mathrm{MHz}, \mathrm{CDCl}_{3}\right): \delta 7.24$ (d, $381 \mathrm{~J}=8.3 \mathrm{~Hz}, 2 \mathrm{H}), 7.08-7.01(\mathrm{~m}, 2 \mathrm{H}), 6.87(\mathrm{~d}, J=8.3 \mathrm{~Hz}, 2 \mathrm{H}), 6.66-$ $3826.60(\mathrm{~m}, 1 \mathrm{H}), 6.49(\mathrm{~s}, 1 \mathrm{H}), 6.36(\mathrm{~s}, 1 \mathrm{H}), 4.98(\mathrm{~d}, J=14.7 \mathrm{~Hz}, 1 \mathrm{H}), 4.34$ $383(\mathrm{~d}, J=14.7 \mathrm{~Hz}, 1 \mathrm{H}), 4.25-4.10(\mathrm{~m}, 1 \mathrm{H}), 3.86-3.75(\mathrm{~m}, 6 \mathrm{H}), 3.43(\mathrm{~s}$, $3843 \mathrm{H}), 3.36-3.25(\mathrm{~m}, 1 \mathrm{H}), 2.96-2.86(\mathrm{~m}, 2 \mathrm{H}), 2.32(\mathrm{~s}, 3 \mathrm{H}) .{ }^{13} \mathrm{C}$ NMR $385\left(75 \mathrm{MHz}, \mathrm{CDCl}_{3}\right): \delta 171.6,159.0,148.4,146.8,141.9,134.1,131.7$, 386 129.7, 129.4, 128.3, 127.5, 125.7, 124.3, 123.7, 114.8, 114.0, 112.1, 55.7, $38755.5,55.3,47.5,44.5,31.7,21.6$. HRMS (EI): $m / z[\mathrm{M}]^{+}$calcd for $388 \mathrm{C}_{26} \mathrm{H}_{27} \mathrm{NO}_{4} \mathrm{~S}$ 449.1661, found 449.1648. Further elution provided $389 \mathrm{~N}$-(4,5-dimethoxy-2-(thiophene-3-yl)phenethyl)-N-(4-methoxybenzyl)but390 2-ynamide $(\mathbf{5 j})$ as a $\sim 3: 2$ mixture of rotamers $\left(t_{\mathrm{R}}=39 \mathrm{~min}\right)$. Yield: $39124 \mathrm{mg}, 27 \%$. Yellow oil. ${ }^{1} \mathrm{H}$ NMR $\left(300 \mathrm{MHz}, \mathrm{CDCl}_{3}\right): \delta 7.44-7.34(\mathrm{~m}$, $3921 \mathrm{H}), 7.17-7.10(\mathrm{~m}, 1 \mathrm{H}), 7.10-7.04(\mathrm{~m}, 1 \mathrm{H}), 7.03-6.94(\mathrm{~m}, 2 \mathrm{H})$, $3936.87-6.73(\mathrm{~m}, 3.6 \mathrm{H}), 6.67(\mathrm{~s}, 0.4 \mathrm{H}), 4.39(\mathrm{~s}, 1.2 \mathrm{H}), 4.25(\mathrm{~s}, 0.8 \mathrm{H}), 3.90$ $394(\mathrm{~s}, 1.8 \mathrm{H}), 3.89(\mathrm{~s}, 1.2 \mathrm{H}), 3.86(\mathrm{~s}, 1.2 \mathrm{H}), 3.85(\mathrm{~s}, 1.8 \mathrm{H}), 3.80(\mathrm{~s}, 1.8 \mathrm{H})$, $3953.78(\mathrm{~s}, 1.2 \mathrm{H}), 3.52-3.41(\mathrm{~m}, 0.8 \mathrm{H}), 3.35-3.23(\mathrm{~m}, 1.2 \mathrm{H}), 2.91-2.73$ $396(\mathrm{~m}, 2 \mathrm{H}), 1.98(\mathrm{~s}, 1.8 \mathrm{H}), 1.96(\mathrm{~s}, 1.2 \mathrm{H}) .{ }^{13} \mathrm{C} \mathrm{NMR}\left(75 \mathrm{MHz}, \mathrm{CDCl}_{3}\right): \delta$ $397159.2,159.0,154.7,154.6,148.5,148.4,147.5,147.2,141.55,141.54$, $398129.6,129.3,129.14,129.10,128.9,128.80,128.77,128.73,128.5,128.4$, $399125.5,125.3,122.6,122.5,114.0,113.9,113.5,113.3,112.9,112.8$, $40088.9,88.6,73.6,73.5,56.1,56.00,55.98,55.95,55.30,55.26,52.2$, $40149.1,46.5,45.2,31.9,30.3,4.06,4.05$. HRMS (EI): $m / z[\mathrm{M}]^{+}$calcd for $402 \mathrm{C}_{26} \mathrm{H}_{27} \mathrm{NO}_{4} \mathrm{~S}$ 449.1661, found 449.1670 .

403 (E)-1-((E)-Hept-3-en-2-ylidene)-7,8-dimethoxy-3-(4-methoxyben$404 \mathrm{zy}($ )-4,5-dihydro-1H-benz[d]azepin-2(3H)-one (4k). The material 405 obtained after workup was subjected to column chromatography on 406 silica gel with heptane/EtOAc $(4: 1 \rightarrow 7: 3)$ as eluent to deliver pure $407 \mathbf{4 k}$. Last fractions containing $4 \mathbf{k}$ overlapping with other impurities were 408 concentrated separately and resubjected to column chromatography to 409 deliver a second portion of pure $4 \mathbf{k}$. Combined yield: $45 \mathrm{mg}, 52 \%$. Pale 410 yellow oil. ${ }^{1} \mathrm{H}$ NMR $\left(300 \mathrm{MHz}, \mathrm{CDCl}_{3}\right): \delta 7.21(\mathrm{~d}, J=8.5 \mathrm{~Hz}, 2 \mathrm{H})$, $4116.86(\mathrm{~d}, J=8.5 \mathrm{~Hz}, 2 \mathrm{H}), 6.74(\mathrm{~s}, 1 \mathrm{H}), 6.53(\mathrm{~s}, 1 \mathrm{H}), 6.36(\mathrm{dt}, J=15.6$, $4121.2 \mathrm{~Hz}, 1 \mathrm{H}), 5.92(\mathrm{dt}, J=15.6,7.0 \mathrm{~Hz}, 1 \mathrm{H}), 4.93(\mathrm{~d}, J=14.7 \mathrm{~Hz}, 1 \mathrm{H})$, $4134.29(\mathrm{~d}, J=14.7 \mathrm{~Hz}, 1 \mathrm{H}), 4.05-3.92(\mathrm{~m}, 1 \mathrm{H}), 3.88-3.75(\mathrm{~m}, 9 \mathrm{H})$, $4143.26-3.13(\mathrm{~m}, 1 \mathrm{H}), 2.89-2.75(\mathrm{~m}, 2 \mathrm{H}), 2.14-1.96(\mathrm{~m}, 5 \mathrm{H}), 1.46-$ $4151.31(\mathrm{~m}, 2 \mathrm{H}), 0.87(\mathrm{t}, J=7.4 \mathrm{~Hz}, 3 \mathrm{H}) .{ }^{13} \mathrm{C} \mathrm{NMR}\left(75 \mathrm{MHz}, \mathrm{CDCl}_{3}\right): \delta$ 416 172.1, 159.0, 148.4, 146.5, 134.0, 133.9, 133.2, 129.8, 129.34, 129.28, 417 128.0, 125.1, 115.4, 114.0, 112.5, 55.9, 55.8, 55.3, 47.4, 44.4, 35.3, 31.7, $41822.5,16.3$, 13.7. HRMS (EI): $\mathrm{m} / z[\mathrm{M}]^{+}$calcd for $\mathrm{C}_{27} \mathrm{H}_{33} \mathrm{NO}_{4}$ 419 435.2410, found 435.2415.

420 (E)-7,8-Dimethoxy-3-(4-methoxybenzyl)-1-(1-phenylpropyli421 dene)-4,5-dihydro-1H-benz[d]azepin-2(3H)-one (4m). An increased $422 \mathrm{Pd}\left(\mathrm{PPh}_{3}\right)_{2} \mathrm{Cl}_{2}(5.6 \mathrm{mg}, 4 \mathrm{~mol} \%)$ loading and extended reaction time 423 of $25 \mathrm{~min}$ were used. The material obtained after workup was 424 subjected to column chromatography on silica gel with heptane/ 425 EtOAc (1:1) as eluent followed by reversed-phase preparative HPLC with gradient pump mode, $\mathrm{MeCN} / \mathrm{H}_{2} \mathrm{O}$ with $0.1 \% \mathrm{HCOOH} 426$ (30-40-50-60-60-70-70-100\%, $10 \mathrm{~min}$ intervals) as eluent, and 427 a flow rate of $10 \mathrm{~mL} / \mathrm{min}$ to deliver pure $4 \mathrm{~m}\left(t_{\mathrm{R}}=39 \mathrm{~min}\right)$. Yield: 428 $45 \mathrm{mg}, 49 \%$. White solid. Mp: 191-193 ${ }^{\circ} \mathrm{C} .{ }^{1} \mathrm{H}$ NMR (300 MHz, 429 $\left.\mathrm{CDCl}_{3}\right): \delta 7.30-7.04(\mathrm{~m}, 8 \mathrm{H}), 6.88(\mathrm{~d}, J=8.3 \mathrm{~Hz}, 2 \mathrm{H}), 6.43(\mathrm{~s}, 1 \mathrm{H}), 430$ $6.18(\mathrm{~s}, 1 \mathrm{H}), 5.05(\mathrm{~d}, J=14.7 \mathrm{~Hz}, 1 \mathrm{H}), 4.37-4.18(\mathrm{~m}, 2 \mathrm{H}), 3.81(\mathrm{~s}, 431$ $3 \mathrm{H}), 3.77(\mathrm{~s}, 3 \mathrm{H}), 3.39-3.25(\mathrm{~m}, 4 \mathrm{H}), 3.14-3.01(\mathrm{~m}, 1 \mathrm{H}), 2.97-2.87432$ $(\mathrm{m}, 2 \mathrm{H}), 2.68-2.53(\mathrm{~m}, 1 \mathrm{H}), 1.60(\mathrm{~s}, 2 \mathrm{H}), 0.92(\mathrm{t}, J=7.4 \mathrm{~Hz}, 3 \mathrm{H}) ; 433$ ${ }^{13} \mathrm{C}$ NMR $\left(75 \mathrm{MHz}, \mathrm{CDCl}_{3}\right): \delta 171.6,159.0,147.8,146.4,143.7,434$ $140.3,134.3,129.7,129.4,129.2,128.0,127.6,126.7,125.6,115.3,435$ 114.0, 111.9, 55.6, 55.32, 55.30, 47.5, 44.6, 31.8, 28.2, 12.4; HRMS 436 (EI): $m / z[\mathrm{M}]^{+}$calcd for $\mathrm{C}_{29} \mathrm{H}_{31} \mathrm{NO}_{4} 457.2253$, found 457.2251. 437

1-(Diphenylmethylene)-7,8-dimethoxy-3-(4-methoxybenzyl)-4,5- 438 dihydro-1H-benz[d]azepin-2(3H)-one (4o). The material obtained 439 after workup was subjected to column chromatography on silica 440 gel with heptane/EtOAc (1:1) as eluent followed by reversed-phase 441 preparative HPLC with gradient pump mode, $\mathrm{MeCN} / \mathrm{H}_{2} \mathrm{O}$ with $0.1 \% 442$ $\mathrm{HCOOH}$ (50-60-70-80-80-90-100\%, 10 min intervals) as eluent, 443 and a flow rate of $10 \mathrm{~mL} / \mathrm{min}(8 \mathrm{~mL} / \mathrm{min}$ for two first intervals $)$ to 444 deliver pure 4o $\left(t_{\mathrm{R}}=24 \mathrm{~min}\right)$. Yield: $15 \mathrm{mg}, 15 \%$. White amorphous 445 solid; ${ }^{1} \mathrm{H}$ NMR (300 MHz, $\left.\mathrm{CDCl}_{3}\right): \delta 7.57-7.46(\mathrm{~m}, 2 \mathrm{H}), 7.42-7.32446$ $(\mathrm{m}, 3 \mathrm{H}), 7.16-7.00(\mathrm{~m}, 5 \mathrm{H}), 6.69-6.59(\mathrm{~m}, 4 \mathrm{H}), 6.53(\mathrm{~s}, 1 \mathrm{H}), 6.37(\mathrm{~s}, 447$ $1 \mathrm{H}), 5.05(\mathrm{~d}, J=14.9 \mathrm{~Hz}, 1 \mathrm{H}), 4.32-4.17(\mathrm{~m}, 1 \mathrm{H}), 3.87(\mathrm{~d}, J=14.9448$ $\mathrm{Hz}, 1 \mathrm{H}), 3.82$ (s, 3H), 3.76 (s, 3H), 3.41 (s, 3H), 3.25-3.96 (m, 3H); 449 ${ }^{13} \mathrm{C}$ NMR $\left(75 \mathrm{MHz}, \mathrm{CDCl}_{3}\right): \delta 170.4,158.7,148.3,146.9,140.71,450$ $140.66,140.63,136.4,130.1,129.7,129.3,128.8,127.93,127.85,127.8,451$ $127.3,126.8,125.5,114.3,113.8,112.2,55.7,55.5,55.2,46.9,44.4,452$ 31.8; HRMS (ESI): $m / z[\mathrm{M}+\mathrm{H}]^{+}$calcd for $\mathrm{C}_{33} \mathrm{H}_{32} \mathrm{NO}_{4} 506.2326,453$ found 506.2318. Further elution provided N-(2-(4,5-dimethoxybiphenyl- 454 2-yl)ethyl)-N-(4-methoxybenzyl)-3-phenylpropiolamide (5o) as a $\sim 1: 1455$ mixture of rotamers $\left(t_{\mathrm{R}}=31 \mathrm{~min}\right)$. Yield: $6 \mathrm{mg}$, $6 \%$. Pale yellow oil. ${ }^{1} \mathrm{H} 456$ NMR $\left(300 \mathrm{MHz}, \mathrm{CDCl}_{3}\right): \delta 7.54-7.24(\mathrm{~m}, 10 \mathrm{H}), 7.01-6.89(\mathrm{~m}, 2 \mathrm{H}), 457$ 6.85-6.74 (m, 2.5H), 6.72 (s, 0.5H), $6.71(\mathrm{~s}, 0.5 \mathrm{H}), 6.67(\mathrm{~s}, 0.5 \mathrm{H}), 458$ $4.39(\mathrm{~s}, 1 \mathrm{H}), 4.24(\mathrm{~s}, 1 \mathrm{H}), 3.92(\mathrm{~s}, 1.5 \mathrm{H}), 3.85(\mathrm{~s}, 1.5 \mathrm{H}), 3.83(\mathrm{~s}, 1.5 \mathrm{H}), 459$ $2 \times 3.79(\mathrm{~s}, 1.5 \mathrm{H}), 3.63(\mathrm{~s}, 1.5 \mathrm{H}), 3.56-3.44(\mathrm{~m}, 1 \mathrm{H}), 3.37-3.25(\mathrm{~m}, 460$ $1 \mathrm{H}), 2.95-2.74(\mathrm{~m}, 2 \mathrm{H}) .{ }^{13} \mathrm{C} \mathrm{NMR}\left(75 \mathrm{MHz}, \mathrm{CDCl}_{3}\right): \delta 159.2,159.0,461$ $154.5,154.4,148.33,148.30,147.4,147.2,141.4,141.3,134.6,134.3,462$ $132.4,132.2,130.04,130.01,129.8,129.5,129.4,128.9,128.7,128.6,463$ $128.5,128.4,128.31,128.27,128.24,127.6,127.1,126.9,120.6,120.5,464$ $114.0,113.9,113.4,113.3,112.8,112.6,90.0,89.7,81.9,81.8,56.1,55.9,465$ 55.7, 55.29, 55.27, 52.3, 49.3, 46.8, 45.4, 32.0, 30.1. HRMS (EI): $\mathrm{m} / z 466$ $[\mathrm{M}]^{+}$calcd for $\mathrm{C}_{33} \mathrm{H}_{31} \mathrm{NO}_{4} 505.2253$, found 505.2283.

467

(E)-3-Methyl-1-(1-phenylethylidene)-4,5-dihydro-1H-benz[d]- 468 azepin-2(3H)-one (4q). The material obtained after workup was 469 subjected to column chromatography on silica gel with heptane/ 470 EtOAc (1:1) as eluent followed by reversed-phase preparative HPLC 471 with gradient pump mode, $\mathrm{MeCN} / \mathrm{H}_{2} \mathrm{O}$ with $0.1 \% \mathrm{HCOOH}(50-472$ $60-70-80-80-90-100 \%, 10 \mathrm{~min}$ intervals) as eluent, and a flow rate 473 of $10 \mathrm{~mL} / \mathrm{min}(8 \mathrm{~mL} / \mathrm{min}$ for two first intervals) to deliver pure $4 \mathbf{q} 474$ $\left(t_{\mathrm{R}}=14 \mathrm{~min}\right)$. Yield: $38 \mathrm{mg}, 69 \%$. White amorphous solid. ${ }^{1} \mathrm{H}$ NMR 475 $\left(300 \mathrm{MHz}, \mathrm{CDCl}_{3}\right): \delta 7.21-6.97(\mathrm{~m}, 7 \mathrm{H}), 6.82-6.68(\mathrm{~m}, 2 \mathrm{H}), 4.55-476$ $4.39(\mathrm{~m}, 1 \mathrm{H}), 3.40-3.06(\mathrm{~m}, 6 \mathrm{H}), 2.27(\mathrm{~s}, 3 \mathrm{H}) .{ }^{13} \mathrm{C} \mathrm{NMR}(75 \mathrm{MHz}, 477$ $\left.\mathrm{CDCl}_{3}\right): \delta 171.6,141.5,137.8,135.3,134.9,133.7,132.6,129.9,128.8,478$ 127.9, 127.0, 126.8, 125.8, 47.7, 32.8, 31.5, 22.1. HRMS $\left(\mathrm{ESI}^{+}\right): \mathrm{m} / z 479$ $[\mathrm{M}+\mathrm{H}]^{+}$calcd for $\mathrm{C}_{19} \mathrm{H}_{20} \mathrm{NO}^{+} 278.1539$, found 278.1534 .

480

(E)-3-Isopropyl-1-(1-phenylethylidene)-4,5-dihydro-1H-benz[d]- 481 azepin-2(3H)-one (4s). The material obtained after workup was 482 subjected to column chromatography on silica gel with heptane/EtOAc 483 (7:3) as eluent followed by reversed-phase preparative HPLC with 484 gradient pump mode, $\mathrm{MeCN} / \mathrm{H}_{2} \mathrm{O}$ with $0.1 \% \mathrm{HCOOH}(30-40-50-485$ $60-60-70-70-100 \%, 10 \mathrm{~min}$ intervals) as eluent, and a flow rate 486 of $10 \mathrm{~mL} / \mathrm{min}$ to deliver pure $4 \mathrm{~s}\left(t_{\mathrm{R}}=33 \mathrm{~min}\right)$. Yield: $27.5 \mathrm{mg}, 45 \% .487$ White amorphous solid. ${ }^{1} \mathrm{H}$ NMR $\left(300 \mathrm{MHz}, \mathrm{CDCl}_{3}\right): \delta 7.19-6.96488$ $9 \mathrm{~m}, 7 \mathrm{H}), 6.82-6.67(\mathrm{~m}, 2 \mathrm{H}), 4.92$ (sept, $J=6.8 \mathrm{~Hz}, 1 \mathrm{H}), 4.24-4.08489$ (m, 1H), 3.58-3.45 (m, 1H), 3.29-2.98 (m, 2H), $2.23(\mathrm{~s}, 3 \mathrm{H}), 1.23490$ $(\mathrm{d}, J=6.8 \mathrm{~Hz}, 6 \mathrm{H}) \cdot{ }^{13} \mathrm{C}$ NMR $\left(75 \mathrm{MHz}, \mathrm{CDCl}_{3}\right): \delta 171.3,141.6,491$ $137.3,135.7,135.2,133.9,132.3,129.8,128.8,127.9,127.0,126.7,492$ 125.7, 43.6, 39.1, 34.5, 21.9, 20.8, 20.6. HRMS (EI): $m / z[\mathrm{M}]^{+}$calcd for 493 $\mathrm{C}_{21} \mathrm{H}_{23} \mathrm{NO} 305.1780$, found 305.1773 .

494 
495 (E)-8,9-Dimethoxy-3-(4-methoxybenzyl)-1-(1-phenylethylidene)496 3,4,5,6-tetrahydrobenzo[d]azocin-2(1H)-one (4u). The material 497 obtained after workup was subjected to column chromatography on 498 silica gel with heptane/EtOAc $(7: 3 \rightarrow 1: 1)$ as eluent followed by 499 reversed-phase preparative HPLC with gradient pump mode, $\mathrm{MeCN}$ / $500 \mathrm{H}_{2} \mathrm{O}$ with $0.1 \% \mathrm{HCOOH}(30-40-50-60-60-70-70-100 \%, 10 \mathrm{~min}$ 501 intervals) as eluent, and a flow rate of $10 \mathrm{~mL} / \mathrm{min}$ to deliver pure $4 \mathbf{u}$ $502\left(t_{\mathrm{R}}=36 \mathrm{~min}\right)$. Yield: $12 \mathrm{mg}, 13 \%$. White amorphous solid; ${ }^{1} \mathrm{H}$ NMR $503\left(300 \mathrm{MHz}, \mathrm{CDCl}_{3}\right): \delta 7.23-7.10(\mathrm{~m}, 6 \mathrm{H}), 6.88-6.80(\mathrm{~m}, 3 \mathrm{H}), 6.47(\mathrm{~s}$, $5043 \mathrm{H}), 4.49(\mathrm{bs}, 2 \mathrm{H}), 3.84-3.74(\mathrm{~m}, 8 \mathrm{H}), 3.58-3.49(\mathrm{~m}, 2 \mathrm{H}), 2.67-2.57$ $505(\mathrm{~m}, 2 \mathrm{H}), 2.24-2.14(\mathrm{~m}, 3 \mathrm{H}), 1.76-1.57(\mathrm{~m}, 4 \mathrm{H}) ;{ }^{13} \mathrm{C} \mathrm{NMR}(75 \mathrm{MHz}$, $\left.506 \mathrm{CDCl}_{3}\right): \delta 172.6,159.0,147.9,147.0,140.6,134.9,133.4,131.9,129.72$, $507129.69,129.6,127.9,127.6,126.9,114.2,113.9,112.8,55.9,55.7$, $50855.3,49.5,49.1,36.0,28.9,21.2$; HRMS (EI): $m / z[\mathrm{M}]^{+}$calcd for $509 \mathrm{C}_{29} \mathrm{H}_{31} \mathrm{NO}_{4} 457.2253$, found 457.2251. Further elution provided $\mathrm{N}$-(3510 (4,5-dimethoxybiphenyl-2-yl)propyl)-N-(4-methoxybenzyl)but-2-ynamide $511(5 \mathbf{u})$ as a $\sim 1: 1$ mixture of rotamers $\left(t_{\mathrm{R}}=43 \mathrm{~min}\right)$. Yield: $31 \mathrm{mg}, 34 \%$. 512 Colorless oil. ${ }^{1} \mathrm{H}$ NMR $\left(300 \mathrm{MHz}, \mathrm{CDCl}_{3}\right): \delta 7.46-7.30(\mathrm{~m}, 3 \mathrm{H})$, $5137.30-7.21(\mathrm{~m}, 2 \mathrm{H}), 7.10-7.00(\mathrm{~m}, 2 \mathrm{H}), 6.88-6.77(\mathrm{~m}, 2 \mathrm{H}), 6.74(\mathrm{~s}$, $5140.5 \mathrm{H}), 6.73(\mathrm{~s}, 0.5 \mathrm{H}), 6.71(\mathrm{~s}, 0.5 \mathrm{H}), 6.67(\mathrm{~s}, 0.5 \mathrm{H}), 4.46(\mathrm{~s}, 1 \mathrm{H}), 4.32$ $515(\mathrm{~s}, 1 \mathrm{H}), 2 \times 3.90(\mathrm{~s}, 1.5 \mathrm{H}), 2 \times 3.85(\mathrm{~s}, 1.5 \mathrm{H}), 3.80(\mathrm{~s}, 1.5 \mathrm{H}), 3.78(\mathrm{~s}$, $5161.5 \mathrm{H}), 3.34-3.24(\mathrm{~m}, 1 \mathrm{H}), 3.18-3.07(\mathrm{~m}, 1 \mathrm{H}), 2.56-2.39(\mathrm{~m}, 2 \mathrm{H})$, $5172.00(\mathrm{~s}, 1.5 \mathrm{H}), 1.89(\mathrm{~s}, 1.5 \mathrm{H}), 1.70-1.52(\mathrm{~m}, 2 \mathrm{H}) .{ }^{13} \mathrm{C}$ NMR (75 MHz, $\left.518 \mathrm{CDCl}_{3}\right): \delta 159.2,159.0,154.8,154.6,148.1,146.9,146.8,141.7,141.6$, $519134.2,133.9,131.1,130.8,129.5,129.41,129.38,128.9,128.4,128.23$, $520128.17,126.9,126.7,114.0,113.9,113.4,113.3,112.20,112.17,89.0$, $52188.9,73.7,73.4,56.05,56.02,55.96,55.95,55.3,55.2,51.6,47.3,46.2$, $52243.1,30.03,29.97,29.8,28.4,4.1,3.9$. HRMS (EI): $m / z[\mathrm{M}]^{+}$calcd for $523 \mathrm{C}_{29} \mathrm{H}_{31} \mathrm{NO}_{4}$ 457.2253, found 457.2276.

524 (E)-7,8-Dimethoxy-3-(4-methoxybenzyl)-1-(4-phenylbut-3-yn-2525 ylidene)-4,5-dihydro-1H-benz[d]azepin-2(3H)-one (4y). The material 526 obtained after workup was subjected to column chromatography 527 on silica gel with heptane/EtOAc $(7: 3 \rightarrow 3: 2)$ as eluent followed by 528 reversed-phase preparative HPLC with gradient pump mode, $\mathrm{MeCN} /$ $529 \mathrm{H}_{2} \mathrm{O}$ with $0.1 \% \mathrm{HCOOH}(30-40-50-60-70-70-80-90-100 \%$, $53010 \mathrm{~min}$ intervals) as eluent, and a flow rate of $10 \mathrm{~mL} / \mathrm{min}$ to deliver 531 pure $4 y\left(t_{\mathrm{R}}=42 \mathrm{~min}\right)$. Yield: $22.4 \mathrm{mg}, 24 \%$. Yellow oil. ${ }^{1} \mathrm{H}$ NMR $532\left(300 \mathrm{MHz}, \mathrm{CDCl}_{3}\right): \delta 7.34-7.19(\mathrm{~m}, 8 \mathrm{H}), 6.88(\mathrm{~d}, J=8.5 \mathrm{~Hz}, 2 \mathrm{H})$, $5336.53(\mathrm{~s}, 1 \mathrm{H}), 4.95 \mathrm{dd}, J=14.7 \mathrm{~Hz}, 1 \mathrm{H}), 4.32(\mathrm{~d}, J=14.7 \mathrm{~Hz}, 1 \mathrm{H})$, $5344.07-3.94(\mathrm{~m}, 1 \mathrm{H}), 3.86(\mathrm{~s}, 3 \mathrm{H}), 3.81(\mathrm{~s}, 6 \mathrm{H}), 3.32-3.20(\mathrm{~m}, 1 \mathrm{H})$, $5352.97-2.80(\mathrm{~m}, 2 \mathrm{H}), 2.22(\mathrm{~s}, 3 \mathrm{H}) .{ }^{13} \mathrm{C}$ NMR $\left(75 \mathrm{MHz}, \mathrm{CDCl}_{3}\right): \delta$ $536170.4,159.1,148.8,146.4,142.6,131.4,129.5,128.4,128.0,125.2$, 537 123.1, 119.2, 114.3, 114.1, 112.1, 94.2, 90.3, 55.84, 55.80, 55.3, 47.6, $53844.5,31.9,21.0$. HRMS (EI): $m / z[\mathrm{M}]^{+}$calcd for $\mathrm{C}_{30} \mathrm{H}_{29} \mathrm{NO}_{4}$ 539 467.2097, found 467.2075. Further elution provided (E)-1-((Z)-4,6540 diphenylhex-3-en-5-yn-2-ylidene)-7,8-dimethoxy-3-(4-methoxybenzyl)-4,5541 dihydro- $1 H$ - benz $[d]$ azepin-2(3H)-one $(6 a)\left(t_{\mathrm{R}}=63 \mathrm{~min}\right)$. Yield: 11.4 $542 \mathrm{mg}, 10 \%$. Yellow oil. ${ }^{1} \mathrm{H}$ NMR $\left(300 \mathrm{MHz}, \mathrm{CDCl}_{3}\right): \delta 7.62-7.48(\mathrm{~m}$, $5434 \mathrm{H}), 7.43-7.18(\mathrm{~m}, 8 \mathrm{H}), 7.07(\mathrm{~s}, 1 \mathrm{H}), 6.88(\mathrm{~d}, J=8.5 \mathrm{~Hz}, 2 \mathrm{H}), 6.82$ $544(\mathrm{~s}, 1 \mathrm{H}) .6 .55(\mathrm{~s}, 1 \mathrm{H}), 4.99(\mathrm{~d}, J=14.7 \mathrm{~Hz}, 1 \mathrm{H}), 4.31(\mathrm{~d}, J=14.7 \mathrm{~Hz}$, $5451 \mathrm{H}), 4.12-3.97(\mathrm{~m}, 1 \mathrm{H}), 3.87(\mathrm{~s}, 3 \mathrm{H}), 3.81(\mathrm{~s}, 3 \mathrm{H}), 3.74(\mathrm{~s}, 3 \mathrm{H})$, $5463.30-3.20(\mathrm{~m}, 1 \mathrm{H}), 2.98-2.78(\mathrm{~m}, 2 \mathrm{H}), 2.61(\mathrm{~s}, 3 \mathrm{H}) .{ }^{13} \mathrm{C}$ NMR $547\left(75 \mathrm{MHz}_{\mathrm{CDCl}}\right): \delta 171.5,159.1,148.8,146.6,139.6,139.5,134.7$, 548 134.1, 131.2, 129.6, 129.4, 128.6, 128.48, 128.45, 128.3, 127.9, 126.2, $549125.0,123.4,123.2,115.7,114.1,112.6,98.7,88.8,55.9,55.8,55.3$, 550 47.4, 44.3, 31.8, 19.3. HRMS (EI): $m / z[\mathrm{M}]^{+}$calcd for $\mathrm{C}_{38} \mathrm{H}_{35} \mathrm{NO}_{4}$ 551569.2566 , found 569.2596 .

552 (E)-3-Methyl-1-(4-phenylbut-3-yn-2-ylidene)-4,5-dihydro-1H553 benz[d]azepin-2(3H)-one (4z). The material obtained after workup 554 was subjected to column chromatography on silica gel with heptane/ $555 \mathrm{EtOAc}(7: 3)$ as eluent followed by reversed-phase preparative HPLC 556 with gradient pump mode, $\mathrm{MeCN} / \mathrm{H}_{2} \mathrm{O}$ with $0.1 \% \mathrm{HCOOH}(30-$ $55740-50-60-70-70-80-90-100 \%, 10$ min intervals) as eluent, and 558 a flow rate of $10 \mathrm{~mL} / \mathrm{min}$ to deliver pure $4 \mathrm{z}\left(t_{\mathrm{R}}=35 \mathrm{~min}\right)$. Yield: $55911.5 \mathrm{mg}, 19 \%$. Yellow solid. Mp: $144-146{ }^{\circ} \mathrm{C} .{ }^{1} \mathrm{H}$ NMR $(300 \mathrm{MHz}$, $\left.560 \mathrm{CDCl}_{3}\right): \delta 7.79-7.68(\mathrm{~m}, 1 \mathrm{H}), 7.31-7.18(\mathrm{~m}, 7 \mathrm{H}), 7.17-7.09(\mathrm{~m}$, $561 \mathrm{lH}), 4.30-4.14(\mathrm{~m}, 1 \mathrm{H}), 3.33-2.96(\mathrm{~m}, 6 \mathrm{H}), 2.17(\mathrm{~s}, 3 \mathrm{H}) .{ }^{13} \mathrm{C}$ NMR $562\left(75 \mathrm{MHz} \mathrm{CDCl}_{3}\right): \delta 170.3,142.8,135.3,133.2,132.0,131.5,129.9$, $563128.3,128.2,128.1,125.6,123.1,119.9,93.6,90.0,47.6,32.8$, 564 31.6, 20.9. HRMS (EI): $m / z[\mathrm{M}]^{+}$calcd for $\mathrm{C}_{21} \mathrm{H}_{19} \mathrm{NO} 301.1467$, found 301.1489. Further elution provided (E)-1-((Z)-4,6-diphenylhex- 565 3-en-5-yn-2-ylidene)-3-methyl-4,5-dihydro-1 H-benz[d]azepin-2(3 H)-one 566 (6b) $\left(t_{\mathrm{R}}=55 \mathrm{~min}\right)$. Yield: $10.5 \mathrm{mg}, 13 \%$. Yellow solid. Mp: $180-567$ $183{ }^{\circ} \mathrm{C} .{ }^{1} \mathrm{H}$ NMR $\left(300 \mathrm{MHz}, \mathrm{CDCl}_{3}\right): \delta 7.60-7.46(\mathrm{~m}, 4 \mathrm{H}), 7.41-568$ $7.12(\mathrm{~m}, 10 \mathrm{H}), 7.00(\mathrm{~s}, 1 \mathrm{H}), 4.35-4.20(\mathrm{~m}, 1 \mathrm{H}), 3.32-2.95(\mathrm{~m}, 6 \mathrm{H}), 569$ $2.56(\mathrm{~s}, 3 \mathrm{H}) .{ }^{13} \mathrm{C} \mathrm{NMR}\left(75 \mathrm{MHz}, \mathrm{CDCl}_{3}\right): \delta 171.4,139.9,139.6,570$ $135.7,134.6,134.5,133.2,132.9,131.2,130.4,128.5,128.44,128.38$, 571 128.1, 127.8, 126.4, 125.8, 123.5, 123.4, 98.6, 88.9, 47.5, 32.6, 31.5, 572 19.2. HRMS (ESI): $m / z[\mathrm{M}+\mathrm{H}]^{+}$calcd for $\mathrm{C}_{29} \mathrm{H}_{26} \mathrm{NO} 404.2009,573$ found 404.2004.

General Procedure for the Heck-Suzuki Tandem Reaction 575 for the Synthesis of 3-Benzazepines 4l,n,p,r,t and Benzazocine 576 4v. $\mathrm{Pd}\left(\mathrm{PPh}_{3}\right)_{2} \mathrm{Cl}_{2}(4.2 \mathrm{mg}, 3 \mathrm{~mol} \%)$, vinylpotassium trifluoroborate 577 3 p $(40 \mathrm{mg}, 0.3 \mathrm{mmol})$, and propargylamide $1(0.2 \mathrm{mmol})$ were loaded 578 into a microwave instrument vial and dissolved in DMF $(1.8 \mathrm{~mL}) .579$ Then $\mathrm{K}_{3} \mathrm{PO}_{4}(85 \mathrm{mg}, 0.4 \mathrm{mmol})$ was added followed by distilled water 580 $(0.45 \mathrm{~mL})$. The reaction vial was evacuated, flushed with argon, sealed, 581 and irradiated under stirring at the set temperature of $115{ }^{\circ} \mathrm{C}$ for 582 20 min utilizing a maximum power of $100 \mathrm{~W}$. Upon completion of the 583 irradiation time the vial was cooled with a stream of air, and then a 584 fresh portion of $\mathrm{Pd}\left(\mathrm{PPh}_{3}\right)_{2} \mathrm{Cl}_{2}(2.8 \mathrm{mg}, 2 \mathrm{~mol} \%)$ was added. The 585 reaction vial was evacuated, flushed with argon, sealed, and irradiated 586 under stirring at the set temperature of $115{ }^{\circ} \mathrm{C}$ for another $15 \mathrm{~min} .587$ Upon completion of the reaction the vial was cooled with a stream 588 of air. The reaction mixture was diluted with EtOAc $(50 \mathrm{~mL})$, washed 589 with water $(2 \times 50 \mathrm{~mL})$, dried over $\mathrm{MgSO}_{4}$, and concentrated under 590 reduced pressure. The resulting crude material was subjected to the 591 appropriate purification procedure.

(E)-1-(But-3-en-2-ylidene)-7,8-dimethoxy-3-(4-methoxybenzyl)- 593 4,5-dihydro-1H-benz[d]azepin-2(3H)-one (4I). The material obtained 594 after workup was subjected to column chromatography on silica gel 595 with heptane/EtOAc (1:1) as eluent to deliver pure 4l. Yield: $57 \mathrm{mg}$, 596 $72 \%$. Beige amorphous solid. ${ }^{1} \mathrm{H}$ NMR $\left(300 \mathrm{MHz}, \mathrm{CDCl}_{3}\right): \delta 7.21$ (d, 597 $J=8.5 \mathrm{~Hz}, 2 \mathrm{H}), 6.86(\mathrm{~d}, J=8.5 \mathrm{~Hz}, 2 \mathrm{H}), 6.72(\mathrm{~s}, 1 \mathrm{H}), 6.69(\mathrm{dd}, J=598$ $17.4,10.9 \mathrm{~Hz}, 1 \mathrm{H}), 6.53(\mathrm{~s}, 1 \mathrm{H}), 5.44(\mathrm{~d}, J=17.4 \mathrm{~Hz}, 1 \mathrm{H}), 5.20(\mathrm{~d}, J=599$ $10.9 \mathrm{~Hz}, 1 \mathrm{H}), 4.92(\mathrm{~d}, J=14.7 \mathrm{~Hz}, 1 \mathrm{H}), 4.31(\mathrm{~d}, J=14.7 \mathrm{~Hz}, 1 \mathrm{H}), 600$ 4.04-3.91 (m, 1H), 3.87-3.77 (m, 9H), 3.27-3.15 (m, 1H), 2.89- 601 $2.77(\mathrm{~m}, 2 \mathrm{H}), 2.08(\mathrm{~s}, 3 \mathrm{H}) .{ }^{13} \mathrm{C} \mathrm{NMR}\left(75 \mathrm{MHz}, \mathrm{CDCl}_{3}\right): \delta 171.7,602$ $159.0,148.6,146.6,136.6,135.6,133.1,129.6,129.3,128.1,124.6,603$ $116.4,115.3,114.0,112.6,56.0,55.9,55.3,47.4,44.4,31.7,15.4 .604$ HRMS (EI): $m / z[\mathrm{M}]^{+}$calcd for $\mathrm{C}_{24} \mathrm{H}_{27} \mathrm{NO}_{4}$ 393.1940, found 605 393.1943.

(E)-7,8-Dimethoxy-3-(4-methoxybenzyl)-1-(pent-1-en-3-ylidene)- 607 4,5-dihydro-1H-benz[d]azepin-2(3H)-one (4n). The material ob- 608 tained after workup was subjected to column chromatography on silica 609 gel with heptane/EtOAc (1:1) as eluent followed by recrystallization/ 610 washing with diethyl ether to deliver pure $4 \mathrm{n}$. Yield: $65 \mathrm{mg}, 80 \% .611$ Yellow solid. Mp: $164-166^{\circ} \mathrm{C} .{ }^{1} \mathrm{H}$ NMR $\left(300 \mathrm{MHz}, \mathrm{CDCl}_{3}\right): \delta 7.21612$ $(\mathrm{d}, J=8.4 \mathrm{~Hz}, 2 \mathrm{H}), 6.86(\mathrm{~d}, J=8.4 \mathrm{~Hz}, 2 \mathrm{H}), 6.70(\mathrm{~s}, 1 \mathrm{H}), 6.61-6.47613$ $(\mathrm{m}, 2 \mathrm{H}), 5.46(\mathrm{~d}, J=17.5 \mathrm{~Hz}, 1 \mathrm{H}), 5.21(\mathrm{~d}, J=11.0 \mathrm{~Hz}, 1 \mathrm{H}), 4.94(\mathrm{~d}, 614$ $J=14.7 \mathrm{~Hz}, 1 \mathrm{H}), 4.29(\mathrm{~d}, J=14.7 \mathrm{~Hz}, 1 \mathrm{H}), 4.05-3.74(\mathrm{~m}, 11 \mathrm{H}), 615$ $3.27-3.15(\mathrm{~m}, 1 \mathrm{H}), 2.90-2.66(\mathrm{~m}, 3 \mathrm{H}), 2.55-2.40(\mathrm{~m}, 1 \mathrm{H}), 1.14(\mathrm{t}, 616$ $J=7.5 \mathrm{~Hz}, 3 \mathrm{H}) .{ }^{13} \mathrm{C} \mathrm{NMR}\left(75 \mathrm{MHz}, \mathrm{CDCl}_{3}\right): \delta 171.7,159.0,148.6,617$ $146.6,138.8,136.2,133.9,129.7,129.4,128.1,124.8,116.3,115.3,618$ 114.0, 112.6, 56.0, 55.8, 55.3, 47.3, 44.3, 31.7, 22.1, 13.5. HRMS (EI): 619 $m / z[\mathrm{M}]^{+}$calcd for $\mathrm{C}_{25} \mathrm{H}_{29} \mathrm{NO}_{4}$ 407.2097, found 407.2097. 620

(Z)-7,8-Dimethoxy-3-(4-methoxybenzyl)-1-(1-phenylallylidene)- 621 4,5-dihydro-1H-benz[d]azepin-2(3H)-one (4p). The material ob- 622 tained after workup was subjected to column chromatography on 623 silica gel with heptane/EtOAc $(1: 1)$ as eluent followed by reversed- 624 phase preparative HPLC with gradient pump mode, $\mathrm{MeCN} / \mathrm{H}_{2} \mathrm{O} 625$ with $0.1 \% \mathrm{HCOOH}(30-40-50-60-70-70-80-90-100 \%, 10$ min 626 intervals) as eluent, and a flow rate of $10 \mathrm{~mL} / \mathrm{min}$ to deliver pure $4 \mathrm{p} 627$ $\left(t_{\mathrm{R}}=40 \mathrm{~min}\right)$. Yield: $16.4 \mathrm{mg}, 18 \%$. White amorphous solid. ${ }^{1} \mathrm{H}$ NMR 628 $\left(300 \mathrm{MHz}, \mathrm{CDCl}_{3}\right): \delta 7.47-7.28(\mathrm{~m}, 5 \mathrm{H}), 6.97-6.82(\mathrm{~m}, 2 \mathrm{H}), 6.70(\mathrm{~s}, 629$ $4 \mathrm{H}), 6.60(\mathrm{~s}, 1 \mathrm{H}), 5.26(\mathrm{~d}, J=10.9 \mathrm{~Hz}, 1 \mathrm{H}), 4.95(\mathrm{~d}, J=15.8 \mathrm{~Hz}, 2 \mathrm{H}), 630$ 4.15-4.00 (m, 1H), $3.91(\mathrm{~s}, 3 \mathrm{H}), 3.88(\mathrm{~s}, 3 \mathrm{H}), 3.84-3.73(\mathrm{~m}, 4 \mathrm{H}), 631$ $3.21-3.10(\mathrm{~m}, 1 \mathrm{H}), 3.04-2.86(\mathrm{~m}, 2 \mathrm{H}) .{ }^{13} \mathrm{C} \mathrm{NMR}\left(75 \mathrm{MHz}, \mathrm{CDCl}_{3}\right): 632$ $\delta 170.5,158.8,148.9,146.8,138.8,137.5,137.3,135.4,129.7,129.3,633$ $129.2,128.3,127.9,127.4,124.2,120.6,114.8,113.7,112.8,56.1,55.9,634$ 
635 55.3, 46.7, 44.1, 31.6. HRMS (EI): $m / z[\mathrm{M}]^{+}$calcd for $\mathrm{C}_{29} \mathrm{H}_{29} \mathrm{NO}_{4}$ 636 455.2097, found 455.2086.

637 (E)-1-(But-3-en-2-ylidene)-3-methyl-4,5-dihydro-1H-benz[d]638 azepin-2(3H)-one (4r). The material obtained after workup was 639 subjected to column chromatography on silica gel with heptane/ $640 \mathrm{EtOAc}(3: 2)$ as eluent to deliver pure 4r. Yield: $37.7 \mathrm{mg}$, 83\%. Yellow 641 amorphous solid. ${ }^{1} \mathrm{H}$ NMR $\left(300 \mathrm{MHz}, \mathrm{CDCl}_{3}\right): \delta 7.27-7.08(\mathrm{~m}, 4 \mathrm{H})$, $6426.64(\mathrm{dd}, J=17.4,10.9 \mathrm{~Hz}, 1 \mathrm{H}), 5.44(\mathrm{~d}, J=17.4 \mathrm{~Hz}, 1 \mathrm{H}), 5.19(\mathrm{~d}, J=$ $64310.9 \mathrm{~Hz}, 1 \mathrm{H}), 4.26-4.09(\mathrm{~m}, 1 \mathrm{H}), 3.28-2.94(\mathrm{~m}, 6 \mathrm{H}), 2.04(\mathrm{~s}, 3 \mathrm{H})$. ${ }_{644}{ }^{13} \mathrm{C}$ NMR $\left(75 \mathrm{MHz}, \mathrm{CDCl}_{3}\right): \delta 171.6,136.8,135.7,135.4,133.6$, 645 132.7, 132.6, 130.3, 127.8, 125.7, 116.7, 47.6, 32.6, 31.4, 15.4. HRMS 646 (EI): $m / z[\mathrm{M}]^{+}$calcd for $\mathrm{C}_{15} \mathrm{H}_{17} \mathrm{NO} 227.1310$, found 227.1328 .

647 (E)-1-(But-3-en-2-ylidene)-3-isopropyl-4,5-dihydro-1H-benz[d]648 azepin-2(3H)-one (4t). The material obtained after workup was 649 subjected to column chromatography on silica gel with heptane/ 650 EtOAc $(7: 3 \rightarrow 1: 1)$ as eluent to deliver pure $4 t$. Yield: $40.3 \mathrm{mg}, 79 \%$. 651 Pale yellow solid. Mp: $136-138{ }^{\circ} \mathrm{C} .{ }^{1} \mathrm{H}$ NMR $\left(300 \mathrm{MHz}, \mathrm{CDCl}_{3}\right)$ : $652 \delta 7.24-7.08(\mathrm{~m}, 4 \mathrm{H}), 6.63(\mathrm{dd}, J=17.4,10.9 \mathrm{~Hz}, 1 \mathrm{H}), 5.42(\mathrm{dd}, J=$ $65317.4,1.1 \mathrm{~Hz}, 1 \mathrm{H}), 5.17(\mathrm{dd}, J=10.9,1.1 \mathrm{~Hz}, 1 \mathrm{H}), 4.87$ (sept, $J=$ $6546.8 \mathrm{~Hz}, 1 \mathrm{H}), 3.95-3.80(\mathrm{~m}, 1 \mathrm{H}), 3.45-3.34(\mathrm{~m}, 1 \mathrm{H}), 3.16-2.92(\mathrm{~m}$, $6552 \mathrm{H}), 2.00(\mathrm{~s}, 3 \mathrm{H}), 1.19(\mathrm{~d}, J=6.8 \mathrm{~Hz}, 6 \mathrm{H}) .{ }^{13} \mathrm{C} \mathrm{NMR}(75 \mathrm{MHz}$, $\left.656 \mathrm{CDCl}_{3}\right): \delta 171.4,137.6,135.5,133.1,132.8,132.5,130.3,127.7,125.6$, 657 116.4, 43.5, 38.9, 34.4, 20.7, 20.5, 15.1. HRMS (ESI): $m / z[\mathrm{M}+\mathrm{H}]^{+}$ 658 calcd for $\mathrm{C}_{17} \mathrm{H}_{22} \mathrm{NO} 256.1696$, found 256.1697 .

659 (E)-1-(But-3-en-2-ylidene)-8,9-dimethoxy-3-(4-methoxybenzyl)660 3,4,5,6-tetrahydrobenzo[d]azocin-2(1H)-one (4v). The material 661 obtained after workup was subjected to column chromatography on 662 silica gel with heptane/EtOAc $(4: 1 \rightarrow 3: 1)$ as eluent to deliver pure $6634 \mathbf{v}$. Last fractions containing $4 \mathbf{v}$ overlapping with other impurities were 664 concentrated separately and resubjected to column chromatography to 665 deliver a second portion of pure $4 \mathrm{v}$. Combined yield: $48 \mathrm{mg}, 59 \%$. 666 Yellow oil. ${ }^{1} \mathrm{H}$ NMR $\left(300 \mathrm{MHz}, \mathrm{CDCl}_{3}\right): \delta 7.15(\mathrm{~d}, J=8.5 \mathrm{~Hz}, 2 \mathrm{H})$, $6676.86-6.78(\mathrm{~m}, 3 \mathrm{H}), 6.62(\mathrm{~s}, 1 \mathrm{H}), 6.26(\mathrm{dd}, J=17.4,10.8 \mathrm{~Hz}, 1 \mathrm{H})$, $6685.34(\mathrm{~d}, J=17.4,0.8 \mathrm{~Hz}, 1 \mathrm{H}), 5.10(\mathrm{~d}, J=10.8,0.8 \mathrm{~Hz}, 1 \mathrm{H}), 3.88$ (s, $6693 \mathrm{H}), 3.87(\mathrm{~s}, 3 \mathrm{H}), 3.79(\mathrm{~s}, 3 \mathrm{H}), 3.45-3.30(\mathrm{~m}, 2 \mathrm{H}), 2.71-2.59(\mathrm{~m}$, $6702 \mathrm{H}), 1.95(\mathrm{~s}, 3 \mathrm{H}), 1.74-1.56(\mathrm{~m}, 2 \mathrm{H}) .{ }^{13} \mathrm{C}$ NMR $\left(75 \mathrm{MHz}, \mathrm{CDCl}_{3}\right)$ : $671 \delta 172.3,158.9,148.2,147.4,136.9,134.8,132.3,130.6,129.7,129.6$, $672129.2,115.8,113.9,112.9,112.8,56.0,55.9,55.3,49.8,49.3,36.2,29.1$, 673 14.6. HRMS (EI): $m / z[\mathrm{M}]^{+}$calcd for $\mathrm{C}_{25} \mathrm{H}_{29} \mathrm{NO}_{4} 407.2097$, found 674407.2078.

675 Synthesis of (E)-7,8-Dimethoxy-3-(4-methoxybenzyl)-2-phenyl-1676 (1-phenylbutylidene)-2,3,4,5-tetrahydro-1H-benz[d]azepine (4w). $677 \mathrm{Pd}\left(\mathrm{PPh}_{3}\right)_{2} \mathrm{Cl}_{2}(4.2 \mathrm{mg}, 3 \mathrm{~mol} \%)$, phenylboronic acid 3a (36.6 mg, $6780.3 \mathrm{mmol})$, and propargylamine $1 \mathrm{~g}(107 \mathrm{mg}, 0.2 \mathrm{mmol})$ were loaded 679 into a microwave instrument vial and dissolved in DMF $(1.8 \mathrm{~mL})$. 680 Then $\mathrm{K}_{3} \mathrm{PO}_{4}(85 \mathrm{mg}, 0.4 \mathrm{mmol})$ was added followed by distilled 681 water $(0.45 \mathrm{~mL})$. The reaction vial was evacuated, flushed with argon, 682 sealed, and irradiated under stirring at a set temperature of $110^{\circ} \mathrm{C}$ for $68325 \mathrm{~min}$ utilizing a maximum power of $100 \mathrm{~W}$. Upon completion of 684 the irradiation time, the vial was cooled with a stream of air, and then 685 a fresh portion of $\mathrm{Pd}\left(\mathrm{PPh}_{3}\right)_{2} \mathrm{Cl}_{2}(2.8 \mathrm{mg}, 2 \mathrm{~mol} \%)$ was added. The 686 reaction vial was evacuated, flushed with argon, sealed, and irradiated 687 under stirring at the set temperature of $110^{\circ} \mathrm{C}$ for another $15 \mathrm{~min}$. 688 Upon completion of the reaction, the vial was cooled with a stream of 689 air. The reaction mixture was diluted with EtOAc $(50 \mathrm{~mL})$, washed 690 with water $(2 \times 50 \mathrm{~mL})$, dried over $\mathrm{Na}_{2} \mathrm{SO}_{4}$, and concentrated under 691 reduced pressure. The resulting crude material was subjected to 692 column chromatography on silica gel with heptane/EtOAc (23:2 $\rightarrow$ 693 17:3) as eluent followed by reversed-phase preparative HPLC with 694 gradient pump mode, $\mathrm{MeCN} / \mathrm{H}_{2} \mathrm{O}(60-70-80-90-100 \%, 10 \mathrm{~min}$ 695 intervals) as eluent, and a flow rate of $10 \mathrm{~mL} / \mathrm{min}$ to deliver pure $4 \mathrm{w}$ $696\left(t_{\mathrm{R}}=37 \mathrm{~min}\right)$. Yield: $29 \mathrm{mg}, 27 \%$. Yellow oil. ${ }^{1} \mathrm{H}$ NMR $(300 \mathrm{MHz}$, $\left.697 \mathrm{CDCl}_{3}\right): \delta 7.30-7.10(\mathrm{~m}, 7 \mathrm{H}), 7.06-6.92(\mathrm{~m}, 3 \mathrm{H}), 6.91-6.78(\mathrm{~m}$, $6984 \mathrm{H}), 6.47(\mathrm{~s}, 1 \mathrm{H}), 5.66(\mathrm{~s}, 1 \mathrm{H}), 4.94(\mathrm{~s}, 1 \mathrm{H}), 3.82-3.71(\mathrm{~m}, 7 \mathrm{H}), 3.40$ $699(\mathrm{~d}, J=13.7 \mathrm{~Hz}, 1 \mathrm{H}), 3.25(\mathrm{~s}, 3 \mathrm{H}), 3.19-3.06(\mathrm{~m}, 2 \mathrm{H}), 3.05-2.92(\mathrm{~m}$, $7002 \mathrm{H}), 2.76-2.62(\mathrm{~m}, 1 \mathrm{H}), 2.49-2.36(\mathrm{~m}, 1 \mathrm{H}), 1.51-1.35(\mathrm{~m}, 2 \mathrm{H})$, $7010.93(\mathrm{t}, J=7.3 \mathrm{~Hz}, 3 \mathrm{H}) .{ }^{13} \mathrm{C} \mathrm{NMR}\left(75 \mathrm{MHz} \mathrm{CDCl}_{3}\right): \delta 158.4,146.9$, $702145.5,143.4,142.8,141.3,139.2,132.4,131.7,131.0,129.4,129.0$, $703128.0,127.8,127.4,126.5,125.8,117.2,113.5,111.1,69.2,57.9,55.7$, $70455.5,55.2,47.2,36.0,35.2,21.6,14.4$. HRMS (EI): $m / z[\mathrm{M}]^{+}$calcd for
$\mathrm{C}_{36} \mathrm{H}_{39} \mathrm{NO}_{3}$ 533.2930, found 533.2959. Further elution provided $\mathrm{N}$-(2- 705 (4,5-dimethoxybiphenyl-2-yl)ethyl)-N-(4-methoxybenzyl)-1-phenylhex-2- 706 $y$-1-amine $(5 \mathbf{w})$ contaminated with unknown impurities $\left(t_{\mathrm{R}}=40 \mathrm{~min}\right) .707$ Amount of obtained material: $43 \mathrm{mg}$. NMR yield: $35 \% .{ }^{1} \mathrm{H}$ NMR 708 $\left(300 \mathrm{MHz}, \mathrm{CDCl}_{3}\right): \delta 7.53-7.44(\mathrm{~m}, 2 \mathrm{H}), 7.35-7.00(\mathrm{~m}, 10 \mathrm{H}), 6.80709$ $(\mathrm{d}, J=8.5 \mathrm{~Hz}, 2 \mathrm{H}), 6.66(\mathrm{~s}, 1 \mathrm{H}), 6.58(\mathrm{~s}, 1 \mathrm{H}), 4.61(\mathrm{~s}, 1 \mathrm{H}), 3.84(\mathrm{~s}, 710$ $3 \mathrm{H}), 3.82(\mathrm{~s}, 3 \mathrm{H}), 3.78(\mathrm{~s}, 3 \mathrm{H}), 3.57(\mathrm{~d}, J=13.4 \mathrm{~Hz}, 1 \mathrm{H}), 3.32(\mathrm{~d}, J=711$ $13.4 \mathrm{~Hz}, 1 \mathrm{H}), 2.81-2.65(\mathrm{~m}, 1 \mathrm{H}), 2.64-2.44(\mathrm{~m}, 3 \mathrm{H}), 2.40-2.23(\mathrm{~m}, 712$ $2 \mathrm{H}), 1.62(\mathrm{q}, J=7.2 \mathrm{~Hz}, 2 \mathrm{H}), 1.06(\mathrm{t}, J=7.4 \mathrm{~Hz}, 3 \mathrm{H}) .{ }^{13} \mathrm{C} \mathrm{NMR}(75713$ $\left.\mathrm{MHz}, \mathrm{CDCl}_{3}\right): \delta 158.5,147.8,146.7,141.5,140.0,134.2,131.9,130.1,714$ $129.9,129.3,128.2,128.0,127.8,127.0,126.6,113.5,113.1,112.9,88.0,715$ 75.4, 56.2, 55.92, 55.87, 55.3, 54.5, 51.9, 31.5, 22.6, 20.9, 13.7. HRMS 716 (EI): $m / z[\mathrm{M}]^{+}$calcd for $\mathrm{C}_{36} \mathrm{H}_{39} \mathrm{NO}_{3}$ 533.2930, found 533.2930. 717

Synthesis of (E)-1-(Hex-1-en-3-ylidene)-7,8-dimethoxy-3-(4-me- 718 thoxybenzyl)-2-phenyl-2,3,4,5-tetrahydro-1H-benz[d]azepine (4x). 719 $\mathrm{Pd}\left(\mathrm{PPh}_{3}\right)_{2} \mathrm{Cl}_{2}$ (4.2 mg, $3 \mathrm{~mol} \%$ ), vinylpotassium trifluoroborate $3 \mathrm{p} 720$ $(40 \mathrm{mg}, 0.3 \mathrm{mmol})$, and propargylamine $1 \mathrm{~g}(107 \mathrm{mg}, 0.2 \mathrm{mmol})$ were 721 loaded into a microwave instrument vial and dissolved in DMF 722 $(1.8 \mathrm{~mL})$. Then $\mathrm{K}_{3} \mathrm{PO}_{4}(85 \mathrm{mg}, 0.4 \mathrm{mmol})$ was added followed by 723 distilled water $(0.45 \mathrm{~mL})$. The reaction vial was evacuated, flushed 724 with argon, sealed, and irradiated under stirring at the set temperature 725 of $115^{\circ} \mathrm{C}$ for 25 min utilizing a maximum power of $100 \mathrm{~W}$. Upon 726 completion of the irradiation time, the vial was cooled with a stream of 727 air, and then a fresh portion of $\mathrm{Pd}\left(\mathrm{PPh}_{3}\right)_{2} \mathrm{Cl}_{2}(4.2 \mathrm{mg}, 3 \mathrm{~mol} \%)$ was 728 added. The reaction vial was evacuated, flushed with argon, sealed, 729 and irradiated under stirring at the set temperature of $115{ }^{\circ} \mathrm{C}$ for 730 another $15 \mathrm{~min}$. Upon completion of the reaction the vial was cooled 731 with a stream of air. The reaction mixture was diluted with EtOAc 732 $(50 \mathrm{~mL})$, washed with water $(2 \times 50 \mathrm{~mL})$, dried over $\mathrm{Na}_{2} \mathrm{SO}_{4}$, and 733 concentrated under reduced pressure. The resulting crude material was 734 subjected to column chromatography on silica gel with heptane/ 735 EtOAc (17:3) as eluent followed by reversed-phase preparative HPLC 736 with gradient pump mode, $\mathrm{MeCN} / \mathrm{H}_{2} \mathrm{O}$ (50-60-70-80-80-90- 737 $100 \%, 10 \mathrm{~min}$ intervals $)$ as eluent, and a flow rate of $10 \mathrm{~mL} / \mathrm{min}(8738$ $\mathrm{mL} / \mathrm{min}$ for two first intervals) to deliver pure $\mathbf{4 x}\left(t_{\mathrm{R}}=51 \mathrm{~min}\right)$. Yield: 739 $53 \mathrm{mg}, 55 \%$. Yellow oil. ${ }^{1} \mathrm{H}$ NMR $\left(300 \mathrm{MHz}, \mathrm{CDCl}_{3}\right): \delta 7.22-7.04740$ $(\mathrm{m}, 7 \mathrm{H}), 6.80(\mathrm{~d}, J=8.6 \mathrm{~Hz}, 2 \mathrm{H}), 6.63(\mathrm{~s}, 1 \mathrm{H}), 6.21(\mathrm{dd}, J=17.5,741$ $11.0 \mathrm{~Hz}, 1 \mathrm{H}), 6.08(\mathrm{~s}, 1 \mathrm{H}), 5.17(\mathrm{dd}, J=17.5,1.1 \mathrm{~Hz}, 1 \mathrm{H}), 4.89$ (dd, 742 $J=11.0,1.1 \mathrm{~Hz}, 1 \mathrm{H}), 4.79(\mathrm{~s}, 1 \mathrm{H}), 3.90(\mathrm{~s}, 3 \mathrm{H}), 3.78(\mathrm{~s}, 3 \mathrm{H}), 3.70-743$ $3.58(\mathrm{~m}, 4 \mathrm{H}), 3.27(\mathrm{~d}, J=13.8 \mathrm{~Hz}, 1 \mathrm{H}), 3.01-2.78(\mathrm{~m}, 3 \mathrm{H}), 2.73-744$ $2.37(\mathrm{~m}, 3 \mathrm{H}), 1.70-1.40(\mathrm{~m}, 2 \mathrm{H}), 1.049 \mathrm{t}, J=7.4 \mathrm{~Hz}, 3 \mathrm{H}) .{ }^{13} \mathrm{C}$ NMR 745 $\left(75 \mathrm{MHz}, \mathrm{CDCl}_{3}\right): \delta 158.4,147.7,145.7,142.4,141.8,137.0,136.1,746$ $132.2,131.5,129.9,129.4,128.2,127.8,126.7,116.4,113.5,112.7,747$ 111.7, 69.6, 57.8, 56.1, 55.8, 55.2, 47.2, 34.4, 29.8, 22.4, 14.8. HRMS 748 (EI): $m / z[\mathrm{M}]^{+}$calcd for $\mathrm{C}_{32} \mathrm{H}_{37} \mathrm{NO}_{3}$ 483.2773, found 483.2787. 749

\section{ASSOCIATED CONTENT}

750

Supporting Information

751

${ }^{1} \mathrm{H}$ and ${ }^{13} \mathrm{C}$ NMR spectra for $\mathbf{1 b}, 4 \mathbf{a}-\mathbf{z}, 5 \mathbf{g}, \mathbf{j}, \mathbf{o}, \mathbf{u}, \mathbf{w}$, and $\mathbf{6 a}, \mathbf{b} .752$ $\mathrm{X}$-ray crystallographic structures and CIF for $4 \mathbf{m}, \mathbf{t}, \mathbf{z}$ and $\mathbf{6 b} .753$ The Supporting Information is available free of charge on the 754 ACS Publications website at DOI: 10.1021/acs.joc.5b00670. 755

\section{AUTHOR INFORMATION}

\section{Corresponding Authors}

*E-mail: vsevolod@suda.edu.cn.

*E-mail: erik.vandereycken@chem.kuleuven.be.

${ }^{\S}$ College of Chemistry, Chemical Engineering and Materials ${ }_{761}$ Science, Dushu Lake Campus, Soochow University, Suzhou 762 215123, China.

${ }^{\#}$ Centre for Chemical and Pharmaceutical Sciences, Central ${ }_{764}$ University of Punjab, Bathinda 151001, India.

Notes

The authors declare no competing financial interest. 


\section{ACKNOWLEDGMENTS}

769 Support was provided by the Fund for Scientific Research 770 (FWO), Flanders, and by the Research Fund of the University 771 of Leuven (KU Leuven). A.A.P. is grateful to the Erasmus 772 Mundus External Cooperation Window (EMECW) - Triple I 773 for a doctoral scholarship. V.A.P. is grateful to the Erasmus 774 Mundus External Cooperation Window (EMECW) - Triple I 775 for a doctoral scholarship and to the Arenderg Doctoral School 776 for a scholarship to complete Ph.D. studies. K.V.H. thanks the 777 Hercules Foundation (Project No. AUGE/11/029 "3D778 SPACE: 3D Structural Platform Aiming for Chemical 779 Excellence") and the Fund for Scientific Research (FWO), 780 Flanders. We thank Ir. B. Demarsin and Prof. J. Rozenski for 781 valuable help with EI and ESI HRMS, respectively. ESI HRMS 782 was made possible by the support of the Hercules Foundation 783 (Grant No. 20100225-7).

\section{$784 \square$ REFERENCES}

785 (1) (a) Kametani, T.; Fukumoto, K. Heterocycles 1975, 3, 931-1004. 786 (b) Weinstock, J.; Hieble, J. P.; Wilson, J. W. Drugs Future 1985, 10, 787 645-696. (c) Kawase, M.; Saito, S.; Motohashi, N. Int. J. Antimicrob. 788 Agents 2000, 14, 193-201. (d) Donets, P. A. Ph.D. Dissertation, 789 University of Leuven (KU Leuven), 2011. (e) Peshkov, V. A. Ph.D. 790 Dissertation, University of Leuven (KU Leuven), 2013 and references 791 cited therein.

792 (2) (a) Pfister, J. R. Heterocycles 1986, 24, 2099-2103. (b) Pauvert, 793 M.; Collet, S.; Guingant, A. Tetrahedron Lett. 2003, 44, 4203-4206. 794 (c) Soldatenkov, A. T.; Soldatova, S. A.; Mamyrbekova-Bekro, J. A.; 795 Gimranova, G. S.; Malkova, A. V.; Polyanskii, K. B.; Kolyadina, N. M.; 796 Khrustalev, V. N. Chem. Heterocycl. Compd. 2012, 48, 1332-1339. 797 (d) Soeta, T.; Ohgai, T.; Sakai, T.; Fujinami, S.; Ukaji, Y. Org. Lett. 798 2014, 16, 4854-4857. (e) Xiao, T.; Li, L.; Lin, G.; Mao, Z.-w.; Zhou, 799 L. Org. Lett. 2014, 16, 4232-4235.

800 (3) García-López, J.-A.; Saura-Llamas, I.; McGrady, J. E.; Bautista, D.; 801 Vicente, J. Organometallics 2012, 31, 8333-8347.

802 (4) (a) Krull, O.; Wünsch, B. Bioorg. Med. Chem. 2004, 12, 1439803 1451. (b) Wirt, U.; Fröhlich, R.; Wünsch, B. Tetrahedron: Asymmetry 804 2005, 16, 2199-2202. (c) Wirt, U.; Schepmann, D.; Wünsch, B. Eur. J. 805 Org. Chem. 2007, 462-475. (d) Husain, S. M.; Fröhlich, R.; 806 Schepmann, D.; Wünsch, B. J. Org. Chem. 2009, 74, 2788-2793.

807 (5) (a) Yu, Y.; Stephenson, G. A.; Mitchell, D. Tetrahedron Lett. 808 2006, 47, 3811-3814. (b) Zhang, L.; Ye, D.; Zhou, Y.; Liu, G.; Feng, 809 E.; Jiang, H.; Liu, H. J. Org. Chem. 2010, 75, 3671-3677.

810 (6) (a) Fidalgo, J.; Castedo, L.; Dominguez, D. Heterocycles 1994, 39, 811 581-589. (b) Kaoudi, T.; Quiclet-Sire, B.; Seguin, S.; Zard, S. Z. 812 Angew. Chem., Int. Ed. 2000, 39, 731-733.

813 (7) (a) Weinstock, J.; Ladd, D. L.; Wilson, J. W.; Brush, C. K.; Yim, 814 N. C. F.; Galladher, G.; McCarthy, M. E.; Silvestry, J.; Sarau, H. M.; 815 Flaim, K. E.; Ackerman, D. M.; Setler, P. E.; Tobia, A. J.; Hahn, R. A. J. 816 Med. Chem. 1986, 29, 2315-2325. (b) Smith, B. M.; Smith, J. M.; Tsai, 817 J. H.; Schultz, J. A.; Gilson, C. A.; Estrada, S. A.; Chen, R. R.; Park, D. 818 M.; Prieto, E. B.; Gallardo, C. S.; Sengupta, D.; Thomsen, W. J.; 819 Saldana, H. R.; Whelan, K. T.; Menzaghi, F.; Webb, R. R.; Beeley, N. 820 R. A. Bioorg. Med. Chem. Lett. 2005, 15, 1467-1470.

821 (8) Tietze, L. F.; Schimpf, R. Synthesis 1993, 876-880.

822 (9) Tietze, L. F.; Schimpf, R. Chem. Ber. 1994, 127, 2235-2240.

823 (10) For a general review on Pd-catalyzed formal hydroarylations of 824 multiple bonds, see: Cacchi, S. Pure Appl. Chem. 1990, 62, 713-722. 825 (11) (a) Donets, P. A.; Van der Eycken, E. Org. Lett. 2007, 9, 3017826 3020. (b) Donets, P. A.; Goeman, J. L.; Van der Eycken, J.; Robeyns, 827 K.; Van Meervelt, L.; Van der Eycken, E. Eur. J. Org. Chem. 2009, 6, $828793-796$.

829 (12) Peshkov, A. A.; Peshkov, V. A.; Pereshivko, O. P.; Van der 830 Eycken, E. V. Tetrahedron 2015, 71, 3863-3871.

831 (13) Peshkov, V. A.; Pereshivko, O. P.; Donets, P. A.; Mehta, V. P.; 832 Van der Eycken, E. V. Eur. J. Org. Chem. 2010, 4861-4867.
(14) For representative examples, see: (a) Cheung, W. S.; Patch, R. 833 J.; Player, M. R. J. Org. Chem. 2005, 70, 3741-3744. (b) Yanada, R.; 834 Obika, S.; Inokuma, T.; Yanada, K.; Yamashita, M.; Ohta, S.; 835 Takemoto, Y. J. Org. Chem. 2005, 70, 6972-6975. (c) Arthuis, M.; 836 Pontikis, R; Florent, J.-C. J. Org. Chem. 2009, 74, 2234-2237. 837 (d) Couty, S.; Liegault, B.; Meyer, C.; Cossy, J. Tetrahedron 2006, 62, 838 3882-3895. (e) Arcadi, A.; Blesi, F.; Cacchi, S.; Fabrizi, G.; 839 Goggiamani, A.; Marinelli, F. J. Org. Chem. 2013, 78, 4490-4498. 840 (f) Castanheiro, T.; Donnard, M.; Gulea, M.; Suffert, J. Org. Lett. 2014, 841 $16,3060-3063$

(15) Guo, L.-N.; Duan, X.-H.; Hu, J.; Bi, H.-P.; Liu, X.-Y.; Liang, Y.- 843 M. Eur. J. Org. Chem. 2008, 1418-1425.

(16) (a) Yu, H.; Richey, R. N.; Carson, M. W.; Coghlan, M. J. Org. 845 Lett. 2006, 8, 1685-1688. (b) Yu, H.; Richey, R. N.; Mendiola, J.; 846 Adeva, M.; Somoza, C.; May, S. A.; Carson, M. W.; Coghlan, M. J. 847 Tetrahedron Lett. 2008, 49, 1915-1918. (c) Carson, M. W.; Coghlan, 848 M. J. World Patent Application WO2009089312, 2009. (d) Yamamoto, 849 J.; Mori, K.; Era, T.; Nakasato, Y. Uchida, K. World Patent Application 850 WO20101990, 2010.

851

(17) CCDC 1044489 (4m), CCDC 1044492 (4t), CCDC 1044490852 $(4 \mathbf{z})$, and CCDC 1044491 (6b) contain the supplementary crystallo- 853 graphic data for this paper. These data can be obtained free of charge 854 from the Cambridge Crystallographic Data Centre via: www.ccdc.cam. 855 ac.uk/data_request/cif. 